\title{
Scavenging quantum information: Multiple observations of quantum systems
}

\author{
P. Rapčan, ${ }^{1}$ J. Calsamiglia, ${ }^{2}$ R. Muñoz-Tapia, ${ }^{2}$ E. Bagan,,${ }^{2,3,4}$ and V. Bužek ${ }^{1,5}$ \\ ${ }^{1}$ Research Center for Quantum Information, Institute of Physics, Slovak Academy of Sciences, Dúbravská cesta 9, \\ 84511 Bratislava, Slovak Republic \\ ${ }^{2}$ Física Teòrica: Informació i Fenòmens Quàntics, Edifici Cn, Universitat Autònoma de Barcelona, E-08193 Bellaterra (Barcelona), Spain \\ ${ }^{3}$ Department of Physics, Hunter College of the City University of New York, 695 Park Avenue, New York, New York 10021, USA \\ ${ }^{4}$ Physics Department, Brookhaven National Laboratory, Upton, New York 11973, USA \\ ${ }^{5}$ Faculty of Informatics, Masaryk University, Botanická 68a, CZ-602 00 Brno, Czech Republic
}

(Received 26 May 2011; published 19 September 2011)

\begin{abstract}
Given an unknown state of a qudit that has already been measured optimally, can one still extract any information about the original unknown state? Clearly, after a maximally informative measurement, the state of the system collapses into a postmeasurement state from which the same observer cannot obtain further information about the original state of the system. However, the system still encodes a significant amount of information about the original preparation for a second observer who is unaware of the actions of the first one. We study how a series of independent observers can obtain, or can scavenge, information about the unknown state of a system (quantified by the fidelity) when they sequentially measure it. We give closed-form expressions for the estimation fidelity when one or several qudits are available to carry information about the single-qudit state, and we study the classical limit when an arbitrarily large number of observers can obtain (nearly) complete information on the system. In addition to the case where all observers perform most informative measurements, we study the scenario where a finite number of observers estimates the state with equal fidelity, regardless of their position in the measurement sequence and the scenario where all observers use identical measurement apparatuses (up to a mutually unknown orientation) chosen so that a particular observer's estimation fidelity is maximized.
\end{abstract}

DOI: $10.1103 /$ PhysRevA.84.032326

PACS number(s): 03.67.-a, 03.65.Ta

\section{INTRODUCTION}

One of the major questions in the interpretation of quantum mechanics is to assert the reality of the wave function. Despite opinions galore, which also include rejecting the necessity of attributing reality to a quantum state [1], there is a consensus in that all information on the state of a system is contained in the wave function (in the sense that it provides the right outcome probabilities for each conceivable measurement on the system). Since all this information is not accessible by a single measurement and, on top of that, quantum formalism only gives outcome probabilities, the meaning of the wave function traditionally has been associated with an infinite ensemble of identically prepared quantum systems (something that cannot be taken literally but only as a conceptual notion). Ground-breaking experiments with individual quantum systems (see, e.g., Refs. [2-5]) and the advent of quantum information technology have brought the focus to individual systems, away from the infinite ensemble picture.

The inherent limitation of quantum measurements to obtain complete information about a system is intimately linked to the disturbance they cause on the state. Clearly, if a given measurement extracts the maximum information on the state of a system, then the same observer cannot obtain additional information by performing further measurements on the system. This almost tautological statement has the startling consequence that quantum measurements, no matter how cautelous they are, inevitably disturb the state of the system (and thereby erase any information on the original state of the system as far as the same observer is concerned). The question remains: What happens if the second measurement is performed by a second observer who independently aims at gaining information about the original state of the system? Indeed, we will see that a second independent observer, who does not know the precise actions nor the measurement results of the previous observers, can still obtain some information on the original state of the system. In this paper, we will study how this information degrades through a sequence of independent measurements performed by independent observers.

We extend the study to the case where several copies of the unknown state are provided to the observers and, more generally, when several copies of the system are used to collectively encode the unknown single-copy state in more efficient ways. ${ }^{1}$ This will allow us to provide insight on a thorny problem in quantum mechanics, namely, the so-called quantum-toclassical transition [7]. The microscopic world is governed by the rules of quantum mechanics, which often seem to be in sharp contrast with the rules of classical physics that govern the macroscopic world. Since we both observe the classical macroscopic world and believe the quantum description is the correct one, the classical properties of systems have to appear within the quantum description in a consistent fashion. How exactly, quantitatively, do these classical properties emerge? Before attempting to answer this question, it is important to recognize what are the essential features that seem to be so different in the classical and quantum worlds. The problem at hand sheds some light on one of these differential aspects, which is the fragility of the information encoded in quantum states versus the enduring nature of classical information.

\footnotetext{
${ }^{1} \mathrm{~A}$ related restricted problem of multiple observations of quantum clocks, i.e., of an evolving phase reference, has been studied in Ref. [6].
} 
Indeed, the information encoded in a classical system can be accessed by an unlimited number of (careful) observers without degrading while quantum mechanics allows retrieving some amount of information, but this degrades as the number of observers increases. We show that the larger the number of copies of a system, the larger the number of observers who can gain a sizable amount of information encoded in the original state. In other words, the information encoded in large collections of quantum systems of the same type behaves classically, in the sense that it is robust with respect to observations.

The paper is structured as follows: In the next section, we present the simplest case, which will illustrate the main ideas of scavenging before going into more general scenarios. In Sec. III, we discuss general considerations relevant for the rest of the paper. In Sec. IV, we analyze the essential scavenging setting in which each observer maximizes the quality of his or her own estimate. We call this scenario the greedy strategy. We study the cases of: (A) the optimal general encoding and (B) a symmetric encoding of a qudit state into a signal state consisting of $N$ identical copies of the given state. In Sec. V, we study the action of repeated weak measurements on a state. Sections VI and VII are devoted to generalizations of the basic setting in which the information about the encoded state is redistributed among the observers by making use of weak measurements. We first consider what we call the egalitarian setting, where the measurement apparatuses are chosen so as to provide estimates of the same quality for all observers. We then study a complementary scenario, where all observers use the very same apparatus, tailored in such a way that a predesignated privileged observer obtains the best estimate. We present our conclusions in Sec. VIII. Three appendices follow with some technical details used in the main text.

\section{THE SIMPLEST NONTRIVIAL EXAMPLE}

Before introducing the general setting of our problem, let us briefly discuss a simple nontrivial example. Imagine a spin-1/2 particle in a pure state $\psi_{0}=\left(1+\boldsymbol{n}_{0} \cdot \boldsymbol{\sigma}\right) / 2$, polarized in some unknown direction $\boldsymbol{n}_{0},\left|\boldsymbol{n}_{0}\right|=1$. Suppose an observer wishes to estimate the polarization direction. By performing a measurement, the observer obtains an estimate (or guess) $\psi_{1}=\left(1+\boldsymbol{n}_{1} \cdot \boldsymbol{\sigma}\right) / 2$. We take the fidelity $f\left(\psi_{1}, \psi_{0}\right):=\operatorname{Tr}\left[\psi_{0} \psi_{1}\right] \equiv\left(1+\boldsymbol{n}_{0} \cdot \boldsymbol{n}_{1}\right) / 2$ as a figure of merit and quantify the success of the estimation procedure by its average, $F_{1}:=\int f\left(\psi_{1}, \psi_{0}\right) d p\left(\psi_{1}, \psi_{0}\right)$, where $d p\left(\psi_{1}, \psi_{0}\right)=$ $d p\left(\psi_{1} \mid \psi_{0}\right) d p\left(\psi_{0}\right)$ is the probability of the event that the true and the estimated pure states are $\psi_{0}$ and $\psi_{1}$, respectively, and the integral is over all such events.

For a uniform prior probability distribution, corresponding to no prior knowledge about the given pure state $\psi_{0}, d p\left(\psi_{0}\right)=$ $d \psi_{0}=d \boldsymbol{n}_{0}=d \varphi d \theta \sin \theta /(4 \pi)$, an optimal estimation strategy is to use a Stern-Gerlach-like apparatus oriented along any chosen axis $\boldsymbol{n}$ [8,9], i.e., a generalized measurement that can be represented by a positive operator-valued measure (POVM) with two elements $M_{ \pm \boldsymbol{n}}=| \pm \boldsymbol{n}\rangle\langle \pm \boldsymbol{n}|$, corresponding to the guesses $\psi_{1}( \pm \boldsymbol{n})=| \pm \boldsymbol{n}\rangle\langle \pm \boldsymbol{n}|$. Thus,

$$
F_{1}=\sum_{\boldsymbol{n}_{1}= \pm \boldsymbol{n}} \int \operatorname{Tr}\left[\psi_{0} \psi_{1}\right] p\left(\psi_{1} \mid \psi_{0}\right) d \psi_{0}
$$

where the dependence of the states on the Bloch vectors is implicit and the conditional probability is given by the Born rule: $p\left(\psi_{1} \mid \psi_{0}\right)=\operatorname{Tr}\left[M_{\boldsymbol{n}_{1}} \psi_{0}\right]=\left(1+\boldsymbol{n}_{0} \cdot \boldsymbol{n}_{1}\right) / 2$. The average fidelity then reads

$$
F_{1}=\sum_{x_{1}= \pm 1} \int d \boldsymbol{n}_{0}\left(\frac{1+x_{1} \boldsymbol{n}_{0} \cdot \boldsymbol{n}_{1}}{2}\right)^{2},
$$

where the exponent 2 results from identical contributions of the fidelity and the outcome probability. This integral can be computed using $\int n_{i} n_{j} d \boldsymbol{n}=\delta_{i j} / 3$ to obtain the well-known result [10],

$$
F_{1}=\frac{2}{3} \text {. }
$$

Notice that the average fidelity of a random-guessing strategy is $1 / 2<2 / 3$.

If the measurement is von Neumann's (an actual SternGerlach), the system collapses into the guessed state $| \pm \boldsymbol{n}\rangle$ upon measuring. Obviously, from the point of view of the observer, any further measurement on such a collapsed system is useless, as it will not increase the average fidelity of the estimation, Eq. (3) (or the mutual information, for that matter), since it is already maximal. However, from the perspective of a second observer, unaware of the guess $\psi_{1}$ and the orientation $\boldsymbol{n}$ of the first observer's Stern-Gerlach apparatus, the postmeasurement state still contains extractable information about the initial state $\psi_{0}$. Regardless of the first observer's apparatus orientation $\boldsymbol{n}$, of the two possible posterior states, $| \pm \boldsymbol{n}\rangle$, the one closer to the true state $\psi_{0}$ occurs with higher probability. Thus, by measuring the state of the system after the first observation, a second observer will indirectly obtain information about $\psi_{0}$.

Since, from the second observer's point of view, all postmeasurement states are equiprobable, the estimation of the state of the system, now $\left|\boldsymbol{n}_{1}\right\rangle$, is formally the same as the estimation of $\psi_{0}$ by the first observer. Hence, the same kind of POVM provides an optimal strategy to estimate $\left|\boldsymbol{n}_{1}\right\rangle$ with a fidelity of $2 / 3$. Then, it is clear that the second observer obtains an estimate of $\psi_{1}$, which is better than the estimate that one would obtain by random guessing and, as argued above, contains information about $\psi_{0}$. More precisely, let us show the average fidelity for the second observer $F_{2}$ is also larger than 1/2. Similar to Eq. (1), we have

$$
F_{2}=\sum_{n_{2}= \pm \boldsymbol{n}} \int \operatorname{Tr}\left[\psi_{0} \psi_{2}\right] p\left(\psi_{2} \mid \psi_{0}\right) d \psi_{0},
$$

where $p\left(\psi_{2} \mid \psi_{0}\right)$ is now the integrated conditional probability over all possible intermediate outcomes. For the two successive measurements that we have described, $F_{2}$ reads

$$
\begin{aligned}
F_{2}= & \sum_{x_{2}= \pm 1} \int d \boldsymbol{n}_{0} \frac{1+x_{2} \boldsymbol{n}_{2} \cdot \boldsymbol{n}_{0}}{2} \\
& \times \sum_{x_{1}= \pm 1} \int d \boldsymbol{n}_{1} \frac{1+x_{2} x_{1} \boldsymbol{n}_{2} \cdot \boldsymbol{n}_{1}}{2} \frac{1+x_{1} \boldsymbol{n}_{1} \cdot \boldsymbol{n}_{0}}{2} .
\end{aligned}
$$

This can be simplified further to give

$$
F_{2}=\iint d \boldsymbol{n}_{1} d \boldsymbol{n}_{0} \frac{1+\left(\boldsymbol{n}_{0} \cdot \boldsymbol{n}_{2}\right)\left(\boldsymbol{n}_{2} \cdot \boldsymbol{n}_{1}\right)\left(\boldsymbol{n}_{1} \cdot \boldsymbol{n}_{0}\right)}{2}=\frac{5}{9},
$$

where, again, we have used the relation $\int n_{i} n_{j} d \boldsymbol{n}=\delta_{i j} / 3$. We note that $F_{2}>1 / 2$, which means that the second observer 
obtains nonzero information about the initial state. Although we have not claimed that a von Neumann measurement is optimal, in Sec. IV, it is proven that this is actually the case and, hence, that the optimal value of the fidelity for the second observer is $F_{2}=5 / 9$.

\section{FORMALISM AND GENERAL CONSIDERATIONS}

In order to make quantitative statements, first, we need to quantify the observers' ability to gain information about the unknown state and, second, to make a judicious precise definition of what we mean by independent observers. Following Refs. [8,10-12], here, we will use, as a figure of merit, the quantum fidelity between unknown input (pure) quantum state $\psi=|\psi\rangle\langle\psi|$ and the estimate, or guess $\psi^{\prime}=\left|\psi^{\prime}\right\rangle\left\langle\psi^{\prime}\right|$ that an observer arrives at, based on his or her measurement outcome: $f\left(\psi, \psi^{\prime}\right)=\left|\left\langle\psi^{\prime} \mid \psi\right\rangle\right|^{2}$. The $k$ th observer's success in gaining knowledge about the original system is given by the mean of the fidelity over all possible input states and guesses,

$$
F_{k}=\int f\left(\psi_{k}, \psi_{0}\right) d p\left(\psi_{k}, \psi_{0}\right),
$$

where $d p\left(\psi_{k}, \psi_{0}\right)=d \psi_{0} d \psi_{k} \tilde{p}\left(\psi_{k} \mid \psi_{0}\right)$ is the joint probability of observer $k$ obtaining the guess $\psi_{k}$ and the original unknown state being $\psi_{0}$, with $\tilde{p}\left(\psi_{k} \mid \psi_{0}\right)$ being the corresponding conditional probability density and assuming a uniform prior distribution for $\psi_{0}$. The integrals run over all joint events, i.e., over the set of all possible pure states $\psi_{0}, \psi_{k} \in \mathcal{S}(\mathcal{H})$.

We want to define a scenario in which, one after the other, each observer gains access to the quantum system but lacks information regarding the actions of the previous observers. In principle, if no further directives are given, each observer will choose a measurement based on the observer's own prior knowledge about it, i.e., the observer may describe the system as the mixed state that results from taking the original input state and performing an average over all the actions that the previous observers might have conceivably performed, which includes, e.g., the obstructive action of resetting the state of the system to a fixed state. We want to find the limits on how well can independent observers recover, or scavenge, information from the very same system after successive measurements. We, therefore, assume that each observer will be careful, i.e., willing to facilitate the task for following observers insofar as this does not conflict with the observer's own priorities. Accordingly, we assume that the observers are free to agree, in advance, on a protocol as long as it does not involve exchanging any information that would allow them to establish a common canonical basis, or reference frame, in which to represent their states and actions-sharing such information would allow all the observers to perform the very same projective measurement and, hence, all of them would obtain the same measurement outcomes and would estimate the original state with equal precision. ${ }^{2}$ Mathematically, imposing this condition is equivalent to describing the actions of the observers in a chosen basis and then averaging the result

\footnotetext{
${ }^{2}$ One can further relax this condition and can allow for forward communication between measurements as long as this is invariant under the choice of basis.
}

over all possible choices. More succinctly, if a given observer performs a quantum operation $\mathcal{L}[\hat{\rho}]$ over the system in a state $\hat{\rho}$, to the other observers, the state will effectively transform as $\mathcal{L}_{s}[\hat{\rho}]=\int d U U \mathcal{L}\left[U^{\dagger} \hat{\rho} U\right] U^{\dagger}$, where $d U$ is the Haar measure of the unitary group acting on the system's Hilbert space.

In order to complete the framework required to present all the results in this paper, we still need to specify what is the figure of merit and what type of information can the observers share when several $(N>1)$ copies of the $d$-dimensional system are available. One option is to consider this multipartite system as a single system and, accordingly, to use the fidelity between the collective input and guess states as a figure of merit and to use the unitaries over the $d^{N}$-dimensional Hilbert space in order to compute the effective transformation $\mathcal{L}_{s}\left[\hat{\rho}^{(N)}\right]$. However, here, we will follow a different, more physically motivated, approach: Since the observers are asked to retrieve information on the encoded single-copy state, we use the fidelity between $d$-dimensional states, and we consider that the observers agree on using the same (mutually unknown) local basis for each of the copies, i.e., the actions of the other observers are known up to a rigid unitary operation $U(g)=$ $g^{\otimes N}, g \in \mathrm{SU}(d)$, i.e., the effective transformation now reads $\mathcal{L}_{s}\left[\hat{\rho}^{(N)}\right]=\int d g U(g) \mathcal{L}\left[U(g)^{\dagger} \hat{\rho}^{(N)} U(g)\right] U(g)^{\dagger}$. This characterization of independent observers is equivalent to that encountered in other protocols, such as aligning reference frames $[13,14]$ where the different parties that do not share a reference frame try to exchange some information [15].

To allow for a further generalization of this scenario, we will introduce the concept of a preparer. The role of the preparer is to encode the state of a single system $\psi_{0}=$ $g\left|\psi_{\text {ref }}\right\rangle\left\langle\psi_{\text {ref }}\left|g^{\dagger},\right| \psi_{\text {ref }}\right\rangle \in \mathcal{H}_{d}, g \in \mathrm{SU}(d)$ into a collective state of the Hilbert space of $N$ systems by a rigid rotation $\Psi=$ $g^{\otimes N}\left|\Psi_{\text {ref }}\right\rangle\left\langle\Psi_{\text {ref }}\left|g^{\dagger \otimes N},\right| \Psi_{\text {ref }}\right\rangle \in \mathcal{H}_{D}, D=d^{N}$, i.e., the signal state $\Psi$ need not be one consisting of $N$ copies of the initial state $\psi_{0}$, but the encoding is still covariant with respect to $\mathrm{SU}(d)$ and its tensor-product representation-covariant, for short. Considering covariant encodings only is actually not a restriction-as we will argue in Sec. IV, this follows from the quantum-operations averaging discussed above.

The computation of the fidelity, Eq. (7), requires the evaluation of the $k$ th observer's conditional probability density $\tilde{p}\left(\psi_{k} \mid \psi_{0}\right)$ for obtaining a guess $\psi_{k}$ given the state to estimate $\psi_{0}$. Naturally, this quantity depends on the preparation (the way $\psi_{0}$ is encoded into a signal state), on the $k$ th observer's measurement and the guessing strategies, and on whatever happened in between. We may decompose each particular conditional joint event into a sum over historiesintermediate events, such as measurement apparatus choices, obtained measurement outcomes, or guesses made based on the outcomes-which may have led to the event $\left(\psi_{k} \mid \psi_{0}\right)$. It is clear that, even though probabilistic strategies in the encodings and measurements choices are possible, these perform equally well as deterministic strategies, which are given by averaging, with their respective probabilities, over those strategies.

Without loss of generality, we may write

$$
\tilde{p}\left(\psi_{k} \mid \psi_{0}\right)=\operatorname{Tr}\left\{\tilde{\mathcal{M}}_{\psi_{k}}^{(k)} \chi_{k-1} \circ \cdots \circ \chi_{1}\left(\varrho_{0}\left(\psi_{0}\right)\right)\right\},
$$


where $\varrho_{0}\left(\psi_{0}\right)$ is the state provided by the preparer, $\chi_{i}$ is a channel induced by an averaged (over all unknowns) measurement of the $i$ th observer, and $\tilde{\mathcal{M}}^{(k)}$ is the operator density, with respect to the measure $d \psi_{k}$ of the POVM $\mathcal{M}^{(k)}$ performed by the $k$ th observer, with measurement outcomes labeled by the guesses $\psi_{k}$ that the observer makes. Note that, should several outcomes lead to the same guess $\psi_{k}$, the POVM element corresponding to $\psi_{k}$ is given by sum of all effects (i.e., POVM elements) of such outcomes.

Equation (8) can also be written as a decomposition over all intermediate observers' guesses $\psi_{i}$,

$$
\begin{aligned}
\tilde{p}\left(\psi_{k} \mid \psi_{0}\right)= & \int d \psi_{k-1} \cdots \int d \psi_{1} \operatorname{Tr}\left\{\tilde { \mathcal { M } } _ { \psi _ { k } } ^ { ( k ) } \left(\tilde{\mathcal{I}}_{\psi_{k-1}}^{(k-1)}\right.\right. \\
& \left.\left.\circ \cdots \circ \tilde{\mathcal{I}}_{\psi_{1}}^{(1)}\right)\left(\varrho_{0}\left(\psi_{0}\right)\right)\right\},
\end{aligned}
$$

where $\tilde{\mathcal{I}}^{(i)}$ is the density of the $i$ th observer's average (over all unknowns) quantum instrument $\mathcal{I}^{(i)}$ with measurement outcomes labeled by the guesses $\psi_{i}$. Quantum instruments, or instruments for short, introduced by Davies and Lewis [16], are the standard mathematical tool used to describe the measurement process when one is interested not only in probabilities of measurement outcomes, but also in the postmeasurement state. An instrument-or an operation-valued measure-assigns, to a set $\mathcal{B}$ of measurement outcomes, an operation $\mathcal{I}_{\mathcal{B}}$ that provides a transformation rule of the state due to the measurement as well as the probability of the outcome, which is given by the trace of the transformed (postmeasurement) state.

In our case, due to the limited mutual knowledge of the observer's (and preparer's) actions, the average instruments $\mathcal{I}^{(i)}$ are covariant with respect to $\mathrm{SU}(d)$ and its symmetric representation $U$, i.e., $\forall \hat{\rho} \in \mathcal{S}\left(\mathcal{H}_{D}\right), \forall g \in \mathrm{SU}(d)$,

$$
\tilde{\mathcal{I}}_{g \psi g^{\dagger}}^{(i)}(\hat{\rho})=U(g) \tilde{\mathcal{I}}_{\psi}^{(i)}\left(U(g)^{\dagger} \hat{\rho} U(g)\right) U(g)^{\dagger} .
$$

For the same reason, the (averaged) encoding $\varrho_{0}$ is covariant with respect to $\mathrm{SU}(d)$ and its symmetric representation $U$, i.e., $\forall \psi \in \mathcal{S}\left(\mathcal{H}_{d}\right), \forall g \in \mathrm{SU}(d)$,

$$
\varrho_{0}\left(g \psi g^{\dagger}\right)=U(g) \varrho_{0}(\psi) U(g)^{\dagger} .
$$

Obviously, the channels in Eq. (8), which are induced by the instruments in Eq. (9), are also covariant with respect to $U$, i.e., $\forall \hat{\rho} \in \mathcal{S}\left(\mathcal{H}_{D}\right), \forall g \in \operatorname{SU}(d)$,

$$
\chi_{i}\left[U(g) \hat{\rho} U(g)^{\dagger}\right]=U(g) \chi_{i}(\hat{\rho}) U(g)^{\dagger} .
$$

Subsequently, the average "encoding" $\chi_{k-1} \circ \cdots \circ \chi_{1}\left(\varrho_{0}(\cdot)\right)$ is also is covariant with respect to $\mathrm{SU}(d)$ and its symmetric representation $U$. The estimation of $\psi_{0}$ can be viewed as the estimation of $\chi_{k-1} \circ \cdots \circ \chi_{1}\left(\varrho_{0}\left(\psi_{0}\right)\right)$ with $\varrho_{0}\left(\psi_{0}\right)$ known to be restricted to the covariant family,

$$
\left\{\varrho_{0}\left(\psi_{0}\right)=U(g) \varrho_{0}\left(\psi_{\mathrm{ref}}\right) U(g)^{\dagger}, g \in \mathrm{SU}(d), U(g)=g^{\otimes N}\right\}
$$

distributed according to the Haar measure $d \mu(g)=d \psi_{0}$. In other words, we have a covariant optimal estimation problem, which, with the fidelity as the cost function, can always be solved by a covariant POVM [17]. Hence, without loss of generality, we may assume the POVM $\mathcal{M}^{(k)}$ to be covariant with respect to $\mathrm{SU}(d)$ and its symmetric representation $U$, i.e., $\forall \psi \in \mathcal{S}\left(\mathcal{H}_{d}\right), \forall g \in \mathrm{SU}(d)$,

$$
\tilde{\mathcal{M}}^{(k)}\left(g \psi g^{\dagger}\right)=U(g) \tilde{\mathcal{M}}^{(k)}(\psi) U(g)^{\dagger} .
$$

Equations (8) and (9) tell us how to proceed further. We search for pairs of covariant encodings $\varrho_{0}$ and $\operatorname{POVM}(\mathrm{s})$ $\mathcal{M}^{(1)}$ fulfilling a desired property of the average fidelity $F_{1}$ (e.g., maximizing $F_{1}$, possibly given additional constraints) for the covariant family of states, Eq. (13). Having at least one such pair $\left\{\varrho_{0}, \mathcal{M}^{(1)}\right\}$, one can evaluate $F_{1}$. Next, for each possible POVM $\mathcal{M}^{(1)}$, obtained in the previous step, consider all covariant quantum instruments $\mathcal{I}^{(1)}$ compatible with the POVM, and calculate the set of covariant channels $\chi_{1}$ that are induced by any of those instruments. Next, search for covariant POVM(s) $\mathcal{M}^{(2)}$ fulfilling a desired property of the average fidelity $F_{2}$ for the average states from the covariant families $\left\{\chi_{1}\left(\varrho_{0}\left(\psi_{0}\right)\right)=U(g) \chi_{1}\left(\varrho_{0}\left(\psi_{\text {ref }}\right)\right) U(g)^{\dagger}, g \in\right.$ $\left.\mathrm{SU}(d), U(g)=g^{\otimes N}\right\}$, distributed as governed by the Haar measure $d \mu(g)$, given by the actions of all channels $\chi_{1}$ from the previous step, and so on.

The task is greatly simplified by the possibility of restricting oneself to covariant apparatuses and channels. If the optimal covariant apparatuses turn out to be unique at each step, the task becomes even simpler. But, even in this case, one still has to calculate the induced channel at each step to obtain the set of average states for the next optimization.

In the following sections, we study various scenarios, which we separate in two groups: Those that require maximally informative measurements, where the action for each observer can be interpreted as a measure-and-prepare channel; and those where the conditions of the problem require that the observers perform weak measurements extracting less information from the system.

\section{GREEDY STRATEGY}

Let us now specialize in the case of greedy observers who primarily want to maximize the fidelity of their own guesses. This is precisely the main scenario that motivates this paper. ${ }^{3}$ Here, the task at hand can be reduced to the problem of a preparer and single observer respectively encoding and estimating quantum states embedded in larger systems. Solutions to the latter problem are often known ( $[8,10,17,19-21])$.

In this scenario, each observer performs the best estimation possible - in other words, there exists no additional measurement that could be performed that would increase the fidelity of the guess, which was obtained based on the original measurement. It follows that the postmeasurement state, after the $i$ th measurement, can depend on the original state $\psi_{0}$ only indirectly, through the obtained guess $\psi_{i}$, hence, the corresponding instrument can be viewed as a measure-andprepare channel. Thus, we can rewrite Eq. (9) in a factorized form

$$
\tilde{p}\left(\psi_{k} \mid \psi_{0}\right)=\int d \psi_{k-1} \tilde{p}\left(\psi_{k} \mid \psi_{k-1}\right) \cdots \int d \psi_{1} \tilde{p}\left(\psi_{2} \mid \psi_{1}\right) \tilde{p}\left(\psi_{1} \mid \psi_{0}\right),
$$

\footnotetext{
${ }^{3}$ Preliminary partial results concerning the greedy scenario have been reported in the proceedings [18].
} 
with

$$
\tilde{p}\left(\psi_{i} \mid \psi_{i-1}\right)=\operatorname{Tr}\left[\tilde{\mathcal{M}}^{(i)}\left(\psi_{i}\right) \varrho_{i-1}\left(\psi_{i-1}\right)\right],
$$

where $\varrho_{i-1}\left(\psi_{i-1}\right)$ is the postmeasurement state after the $(i-$ 1)th measurement given that the obtained guess has been $\psi_{i-1}$.

Using the Bloch-vector formalism, we may rewrite the average fidelity, Eq. (7), as

$$
F_{k}=\frac{1}{d}\left[1+(d-1) \int d \psi_{0} d \psi_{k} \boldsymbol{n}\left(\psi_{k}\right) \cdot \boldsymbol{n}\left(\psi_{0}\right) \tilde{p}\left(\psi_{k} \mid \psi_{0}\right)\right]
$$

where $\boldsymbol{n}(\psi)$ stands for the generalized Bloch vector of a pure state $\psi \in \mathcal{S}\left(\mathcal{H}_{d}\right)$ (see Appendix A for details).

As we argued in Sec. III, the average instrument $\mathcal{I}^{(i)}$ and, thus, the induced POVM $\mathcal{M}^{(i)}$ and the encoding $\varrho_{i}, i<k$, are covariant, while the POVM $\mathcal{M}^{(k)}$ can be chosen to be covariant. Hence, without loss of generality, we may restrict our attention to the optimal covariant POVMs for the set of equiprobable states of the covariant family $\left\{\varrho_{i-1}\left(\psi_{i-1}\right)=U(g) \varrho_{i-1}\left(\psi_{\text {ref }}\right) U(g)^{\dagger}, \psi_{i-1}=\right.$ $\left.g \psi_{\text {ref }} g^{\dagger}, U(g)=g^{\otimes N}, g \in \mathrm{SU}(d)\right\}$.

For such a situation, in Appendix A, we show that

$$
\int d \psi_{i-1} \boldsymbol{n}\left(\psi_{i-1}\right) \tilde{p}\left(\psi_{i} \mid \psi_{i-1}\right)=\Delta_{i} \boldsymbol{n}\left(\psi_{i}\right)
$$

where $\Delta_{i}$ is a number.

Plugging Eqs. (15) and (18) into Eq. (17), we have

$$
\begin{aligned}
F_{k} & =\frac{1}{d}\left(1+(d-1) \prod_{i=1}^{k} \Delta_{i} \int d \psi_{k} \boldsymbol{n}\left(\psi_{k}\right) \cdot \boldsymbol{n}\left(\psi_{k}\right)\right) \\
& =\frac{1}{d}\left(1+(d-1) \prod_{i=1}^{k} \Delta_{i}\right) .
\end{aligned}
$$

Thus, successive maximizations of $F_{1}, F_{2}, \ldots, F_{k}$ are achieved via successive maximizations of $\Delta_{1}, \ldots, \Delta_{k}$. The maximization of $\Delta_{i}$ is over pairs $\varrho_{i-1}, \mathcal{M}_{i}$ for the set of states $\left\{\varrho_{i-1}\left(\psi_{i-1}\right)\right\}$ with unknown, hence, equiprobable previous observer's guess $\psi_{i-1}=\left|\psi_{i-1}\right\rangle\left\langle\psi_{i-1}\right| \in \mathcal{S}\left(\mathcal{H}_{d}\right)$.

If the initial encoding $\varrho_{0}$ has been optimal, then one cannot achieve a better performance than if we take $\forall i, \varrho_{i} \equiv \varrho_{0}$, i.e., $\Delta_{i}=\max \Delta_{1}=: \Delta$. Hence, the maximum $\mathcal{F}_{k}$ of the average fidelity $F_{k}$ if all $F_{i}, i<k$ are, one after another, maximal, reads

$$
\mathcal{F}_{k}=\frac{1}{d}\left[1+(d-1) \Delta^{k}\right]
$$

The situation is different if the initial encoding has been restricted by some additional requirements, e.g., encoding into copies of the state $\psi_{0}$, which turns out to be a suboptimal encoding with $\Delta_{\text {sym }}$. Then, for $i \geqslant 1$, the best strategy is, naturally, to take $\varrho_{i}$ equal to the unrestricted optimal $\varrho_{0}$. That is, for the problem of $k$ greedy observers independently estimating $N$ copies of an unknown state, the fidelity will read

$$
\mathcal{F}_{k}=\frac{1}{d}\left[1+(d-1) \Delta_{\mathrm{sym}} \Delta^{k-1}\right] .
$$

In the following subsections, we give the explicit expressions for the fidelity $\mathcal{F}_{k}$ for some relevant cases, which amounts to solving the single-observer problem (i.e., computing $\Delta$ ).

\section{A. The fidelity for the optimal $N$-qubit encoding}

Here, we treat the optimization of the qubit state encoding with full generality. The optimal $U$-covariant encoding of single-qubit states (or, equivalently, of spatial directions corresponding to spin-1/2 particles) into $N$-qubit states is given by Bagan et al. in Ref. [19].

The optimal signal state $(k=0)$ as well as the state prepared after the $k$ th measurement $k>0$ read

$$
\varrho_{k}\left(\boldsymbol{m}_{k}\right)=U\left(\boldsymbol{m}_{k}\right)|A\rangle\langle A| U^{\dagger}\left(\boldsymbol{m}_{k}\right), \quad k \geqslant 0,
$$

where

$$
|A\rangle=\sum_{j=0}^{N / 2} A_{j}|j, 0\rangle
$$

(for simplicity, we assume that $N$ is even) with the coefficients $A_{j}$ such that $|A\rangle$ is the eigenvector corresponding to the maximal eigenvalue of the matrix

$$
\left(\begin{array}{ccccc}
0 & c_{l-1} & 0 & \ldots & 0 \\
c_{l-1} & \ddots & \ddots & \ddots & \vdots \\
0 & \ddots & \ddots & c_{2} & 0 \\
\vdots & \ddots & c_{2} & 0 & c_{1} \\
0 & \ldots & 0 & c_{1} & 0
\end{array}\right),
$$

where

$$
l=\frac{N}{2}+1,
$$

and

$$
c_{i}=\frac{i}{\sqrt{(2 i+1)(2 i-1)}} .
$$

The operator density of the corresponding optimal measurement can also be found in [19]

$$
M^{(k)}\left(\boldsymbol{m}_{k}\right)=U\left(\boldsymbol{m}_{k}\right)|B\rangle\langle B| U^{\dagger}\left(\boldsymbol{m}_{k}\right), \quad k>1,
$$

where

$$
|B\rangle=\sum_{j=0}^{N / 2} \sqrt{2 j+1}|j, 0\rangle .
$$

In this case,

$$
\Delta=x_{N / 2+1},
$$

where $x_{N / 2+1}$ is the largest zero of the Legendre polynomial $P_{N / 2+1}(x)$. Thus,

$$
\mathcal{F}_{k}^{\mathrm{op}}=\frac{1}{2}\left[1+x_{N / 2+1}^{k}\right] .
$$

Asymptotically, it is known that

$$
x_{n}=1-\frac{\xi_{0}^{2}}{2 n^{2}}+\cdots \text {, }
$$


where $\xi_{0}=2.4$ is the first zero of the Bessel function $J_{0}(x)$. Hence, asymptotically (for $N \rightarrow \infty$ ),

$$
\Delta \cong 1-\frac{2 \xi_{0}^{2}}{N^{2}}
$$

and

$$
\mathcal{F}_{k}^{\mathrm{op}} \cong \frac{1}{2}\left[1+\left(1-\frac{2 \xi_{0}^{2}}{N^{2}}\right)^{k}\right]
$$

In the case where the signal state is given as $N$ copies of the unknown state, i.e., $\psi_{0}^{\otimes N}$, the first $(k=1)$ delta factor needs to be replaced by that of the well-known $N$-qubit pure state estimation $\Delta_{\text {sym }}=\frac{N}{N+2}$ (see the next subsection for general qudit derivation). So that,

$$
\begin{aligned}
\mathcal{F}_{k}^{N \text { copy }} & =\frac{1}{2}\left[1+\frac{N}{N+2} x_{N / 2+1}^{k-1}\right] \\
& \cong \frac{1}{2}\left[1+\left(1-\frac{2}{N}\right)\left(1-\frac{2 \xi_{0}^{2}}{N^{2}}\right)^{k-1}\right]
\end{aligned}
$$

We note that by allowing operations to act on the whole Hilbert space of $N$ qubits provides a significant advantage with respect to strategies relying on the encoding into $N$ copies $\psi_{0}^{\otimes N}$, (which lies in the completely symmetric subspace): In the latter case, the maximum fidelity is approached as $1 / N$, in contrast to the $1 / N^{2}$ behavior found in the optimal case-see the end of this section for a more detailed discussion.

\section{B. The fidelity for $N$-parallel qudits}

For $d$-dimensional states with $d>2$, the general optimization is a hard problem to solve. In this section, we will limit ourselves to the situation where the measurement apparatuses of the different observers are restricted to operate in the Hilbert space of the initial state, which we also take to be $N$ copies of an arbitrary pure qudit state. That is, during the whole measurement sequence, the system will be constrained in the totally symmetric subspace of the state space $\mathcal{S}\left(\mathcal{H}_{D}\right)$. A natural way to impose this limitation could be to require that, if in the fortunate, but extremely rare, event that an observer guesses the input state correctly, then the output state should be left in exactly the same collective state as the input.

The POVM $\mathcal{M}=\mathcal{M}^{\text {sym }}$, optimal for the encoding into copies, is known to be the extremal covariant POVM [17] with the operator density on the relevant symmetric subspace given by

$$
\tilde{\mathcal{M}}^{\mathrm{sym}}(\psi)=d_{N}^{\mathrm{sym}}|\psi\rangle\left\langle\left.\psi\right|^{\otimes N}\right.
$$

where

$$
|\psi\rangle^{\otimes N}=\left(g\left|\psi_{\mathrm{ref}}\right\rangle\right)^{\otimes N}, \quad g \in \mathrm{SU}(d), \quad\left|\psi_{\mathrm{ref}}\right\rangle \in \mathcal{H}_{d}
$$

The maximal single-observation fidelity is

$$
\begin{aligned}
\mathcal{F}_{1}^{\text {sym }}= & \int d \psi d \hat{\psi}\left|\langle\psi \mid \hat{\psi}\rangle^{N}\right|^{2} \tilde{p}(\hat{\psi} \mid \psi) \\
= & d_{N}^{\text {sym }} \int d \psi\left|\left\langle\psi \mid \psi_{\text {ref }}\right\rangle\right|^{2(N+1)} \\
= & d_{N}^{\text {sym }}\left\langle\Psi_{\text {ref }}^{\text {sym }}\right|\left[\int d \mu(g) \mathcal{U}(g)\left|\Psi_{\text {ref }}^{\text {sym }}\right\rangle\left\langle\Psi_{\text {ref }}^{\text {sym }}\right| \mathcal{U}(g)^{\dagger}\right] \\
& \times\left|\Psi_{\text {ref }}^{\text {sym }}\right\rangle=\frac{d_{N}^{\text {sym }}}{d_{N+1}^{\text {sym }}},
\end{aligned}
$$

where $\left|\Psi_{\text {ref }}^{\mathrm{sym}}\right\rangle=\left|\psi_{\text {ref }}\right\rangle^{\otimes(N+1)}$ and the dimension of the completely symmetric representation is given by

$$
d_{N}^{\text {sym }}=\left(\begin{array}{c}
N+d-1 \\
N
\end{array}\right) .
$$

Substituting Eq. (38) into Eq. (37), we get

$$
\mathcal{F}_{1}^{\mathrm{sym}}=\frac{N+1}{N+d} .
$$

Using Eq. (20), we have

$$
\begin{aligned}
\mathcal{F}_{k}^{\mathrm{sym}} & =\frac{1}{d}\left[1+(d-1)\left(\frac{N}{N+d}\right)^{k}\right], \\
& \cong \frac{1}{d}\left[1+(d-1)\left(1-\frac{d}{N}\right)^{k}\right],
\end{aligned}
$$

where the approximation holds in the asymptotic limit of a large number of copies.

From the above results, we can readily obtain some conclusions on how large a system needs to be in order to be considered classical as far as the readout of the encoded information is concerned. The minimum size $N$ is related to the number of independent observations we may perform on it and still get good estimates. For parallel spins, we see that we need a minimum size on the order of

$$
N \sim k^{\alpha}, \quad \alpha>1,
$$

if we wish to obtain the classical behavior,

$$
F_{k} \rightarrow 1 \text {. }
$$

For smaller sizes $N \sim k^{\alpha}$ with $\alpha<1$, the fidelity inevitably drops to that of the random-guessing strategy,

$$
F_{k} \rightarrow \frac{1}{2}
$$

For the optimal recycling of information, we see that, for qubits,

$$
F_{k} \rightarrow 1 \text { if } N \sim k^{\alpha}, \quad \alpha>1 / 2,
$$

hence, in this case, in order for a system of spins to be considered classical, it's size (number of spins) needs to scale as the square root of the number of observations.

Note that the above is not in contradiction with the result [22] where the authors obtain $k=O\left(N^{2}\right)$ for what we call symmetric encoding into parallel spins. The quantity considered in Ref. [22], related to longevity $k$, of a directional reference carried by a quantum system, is the first moment of the spin-projection operator for the state after $k$ uses $\left\langle J_{\boldsymbol{n}(\psi)}\right\rangle_{\rho_{k}(\psi)}=\left(2 F_{k}-1\right)(N+2) / 2$, which they require to stay 
above arbitrary, but fixed, threshold $c$. We require that the threshold approaches $(N+2) / 2$ for all $N$, and we take the limit $N \rightarrow \infty$.

\section{WEAK MEASUREMENTS}

We now aim to generalize the problem for situations where the observers do not pursue the mere maximization of their estimation fidelity but adopt strategies where the information on the unknown state is redistributed in different ways among the various independent observers. In the following sections, we will study the case where $K$ observers estimate the original state with equal, but maximal, fidelity (egalitarian strategy) and the case where all observers use the same measurement apparatus and the goal is to optimize the estimation fidelity of the $k$ th observer.

In both instances, the measurements performed need to be weak, i.e., in general, not extracting all of the extractable information and, hence, inflicting less disturbance to the state. As in the greedy-observers scenario, it suffices to consider covariant measurements. All $U$-covariant POVMs (with outcomes labeled by the guesses) have an operator density of the form

$$
\tilde{\mathcal{M}}(\psi) \sim U(g) S_{\text {ref }} U(g)^{\dagger},
$$

with

$$
\psi=g \psi_{\mathrm{ref}} g^{\dagger}, \quad U(g)=g^{\otimes N}, \quad g \in \mathrm{SU}(d),
$$

where $S_{\text {ref }}$ can be a positive operator commuting with $\left\{U(g) ; g \in G_{\text {ref }}\right\}$, where $G_{\text {ref }} \subset \mathrm{SU}(d)$ is the set of unitaries that leave the reference state $\psi_{\text {ref }}$ invariant [17] — the completeness POVM relation can easily be imposed in this covariant construction.

It is clear that, for optimal weak measurements, the postmeasurement states, in general, will not be pure anymore. They will depend not only on the measurement outcome (guess) of the current observer, but also on particular guesses of all preceding observers and the preparation parameter $\psi_{0}$. The probability density of obtaining a measurement outcome leading to a guess $\psi_{k}$, given the previous observer has obtained the guess $\psi_{k-1}$, is not independent of previous observers' guesses, i.e., $\tilde{p}_{k}\left(\psi_{k} \mid \psi_{k-1}, \ldots, \psi_{0}\right)=\tilde{p}\left(\psi_{k} \mid \psi_{k-1}\right)$ does not hold, in general. Thus, we have to start with the histories decomposition, Eq. (8), since Eq. (9) does not simplify to Eq. (15) anymore.

To proceed further, hence, we will follow the approach outlined in Eq. (8), where to the $k$ th observer, the action of all previous observers is described as covariant channels. We, hence, need to calculate actions of the channels $\chi_{k}, k=$ $1, \ldots, K-1$. We will do that in what follows for the singlequdit case and for the qubit case restricted to encoding into copies.

\section{A. Single copy: Arbitrary dimension}

We start with the case of a (single copy) of an unknown pure state $\psi_{0}=\left|\psi_{0}\right\rangle\left\langle\psi_{0}\right|$ of arbitrary finite dimension $d$. A qudit being measured using a $\mathrm{SU}(d)$-covariant instrument undergoes, if the measurement outcome is unknown, the dynamics given by the channel $\chi_{k}$, which is $\mathrm{SU}(d)$-covariant, i.e., a convex combination of the identity channel and the contraction to the total mixture, acting as

$$
\chi_{k}(\hat{\rho})=r_{k} \hat{\rho}+\left(1-r_{k}\right) \mathbb{1} / d .
$$

On the other hand, the $k$ th observer's fidelity of the guess of an original reference state $\psi_{\text {ref }}$, for an effectively encoded state $\hat{\rho}_{\text {ref }}^{(k-1)}=\chi_{k-1} \circ \cdots \circ \chi_{1}\left(\left|\psi_{\text {ref }}\right\rangle\left\langle\psi_{\text {ref }}\right|\right.$ - the result of sending $\left|\psi_{\text {ref }}\right\rangle\left\langle\psi_{\text {ref }}\right|$ through the $\operatorname{SU}(d)$-covariant channels $\chi_{1}, \ldots, \chi_{k-1}$-is given by

$$
\begin{aligned}
F_{k}= & \sum_{o} \int d \mu(g) \operatorname{Tr}\left[g\left|\psi_{\text {ref }}\right\rangle\left\langle\psi_{\text {ref }}\left|g^{\dagger} g_{o}\right| \psi_{\text {ref }}\right\rangle\left\langle\psi_{\text {ref }}\right| g_{o}^{\dagger}\right] \\
& \times \operatorname{Tr}\left[g \hat{\rho}_{\text {ref }}^{(k-1)} g^{\dagger} M_{o}^{(k)}\right],
\end{aligned}
$$

where the state we wish to estimate is $g\left|\psi_{\text {ref }}\right\rangle\left\langle\psi_{\text {ref }}\right| g^{\dagger}$. For convenience, we assume the (currently) last observer's POVM to be one with finitely many outcomes denoted by $o$. The guess associated with an outcome is denoted by $\psi_{o}=$ $g_{o}\left|\psi_{\text {ref }}\right\rangle\left\langle\psi_{\text {ref }}\right| g_{o}^{\dagger}$. Using Eq. (C1) of Appendix C, we obtain

$$
F_{k}=\frac{\left(d O_{S}^{(k-1)}-1\right) O_{M}^{(k)}}{d(d+1)(d-1)}+\frac{d-O_{S}^{(k-1)}}{(d+1)(d-1)},
$$

where $O_{S}^{(k-1)}$ is the overlap of the states,

$$
O_{S}^{(k-1)}=\operatorname{Tr}\left[\psi_{\text {ref }} \hat{\rho}_{\text {ref }}^{(k-1)}\right],
$$

and $O_{M}^{(k)}$ is the overlap between guesses and corresponding POVM elements,

$$
O_{M}^{(k)}=\sum_{o} \operatorname{Tr}\left[\psi_{o} M_{o}^{(k)}\right] .
$$

For a general $\mathrm{SU}(d)$-covariant qudit channel, Eq. (48), induced by the $k$ th measurement and the averaging due to lack of knowledge about it, one trivially has

$$
\begin{aligned}
F_{k+1}= & r_{k} \sum_{q} \int d g \operatorname{Tr}\left[g\left|\psi_{0}\right\rangle\left\langle\psi_{0}\left|g^{\dagger} g_{q}\right| \psi_{\mathrm{ref}}\right\rangle\left\langle\psi_{\mathrm{ref}}\right| g_{q}^{\dagger}\right] \\
& \times \operatorname{Tr}\left[g \hat{\rho}_{\mathrm{ref}}^{(k-1)} g^{\dagger} M_{q}^{(k+1)}\right]+\frac{1-r_{k}}{d} \\
= & r_{k}\left(F-\frac{1}{d}\right)+\frac{1}{d},
\end{aligned}
$$

where $F$ is the average fidelity of the $(k+1)$ th observer's guess if the measurement would have been performed on the state $\hat{\rho}^{(k-1)}$-i.e., as if the $k$ th observer would not have measured at all. The fidelity has the property $1 / d \leqslant F \leqslant 1$ (the lower bound is provided by random guessing; smaller values can be reached by intentionally bad estimates). It follows that, in order to maximize the possible $F_{k+1}$ for any fixed measurement $M^{(k+1)}$, one has to have $r_{k}$ as large as possible. Naturally, $r_{k}$ will ultimately be limited by the achieved $F_{k}$ but also by the particular choice of the $k$ th observer's measurements (instrument) attaining that value of the fidelity.

At this stage, we have to be more explicit in describing the observers' measurement apparatuses. In particular, we have to specify the instrument realizing the POVM $M^{(k)}$ that appears in Eqs. (49) and (52). Here, we have two options: The first option is that the quantum operation performed, upon obtaining any outcome $o$, is given by a single-term Kraus decomposition. That is, the un-normalized postmeasurement 
state for an outcome $o$ is given by $\hat{\rho}_{\text {post }}=A_{o}^{\dagger} \hat{\rho}_{\text {in }} A_{o}$ (we omit the index indicating the outcome in the postmeasurement states). The second option is that there exist some outcomes for which the operation has multiple Kraus operators in its decomposition $\left(\hat{\rho}_{\text {post }}=\sum_{i=1}^{n} B_{\alpha, i}^{\dagger} \hat{\rho}_{\text {in }} B_{\alpha, i}, n>1, \exists \alpha ; B_{\alpha, i} \neq \hat{0}, \forall i\right)$. In the latter case, we formally redefine the POVMs used in Eqs. (49) and (52)—we simply use the language of the fine-grained measurement with POVM elements $M_{\alpha, i}^{\prime}:=B_{\alpha, i}^{\dagger} B_{\alpha, i}$ and operations defined by $\hat{\rho}_{\text {post }}=B_{\alpha, i}^{\dagger} \hat{\rho}_{\text {in }} B_{\alpha, i}$ for all $\alpha$ 's where a multiterm Kraus decomposition would otherwise take place. Since the additional labels $i$ are not used for anything (they are not accessible to the observer and, thus, cannot influence the guess), these formal apparatuses provide an equivalent description. Thus, we can always assume a description of the measurement process in terms of an apparatus with a single-term Kraus decomposition for each outcome. For such apparatuses, the averaged effective channel is of the form, Eq. (48), with the parameter $r_{k}$ acquiring a particularly simple form (see Appendix C),

$$
r_{k}=\frac{c-1}{(d+1)(d-1)}, \quad \text { where } c=\sum_{o}\left|\operatorname{Tr} A_{o}^{(k)}\right|^{2} .
$$

Recall that we wish to have $r_{k}$ as large as possible given the $k$ th observer's achieved fidelity $F_{k}$, i.e., in the language of the single-Kraus-term apparatuses, the value of $c$ as large as possible.

For a given value of $F_{k}$, a measurement both reaching $F_{k}$, i.e., the required $O_{M}^{(k)}$ in Eq. (50), and maximizing $c$ in Eq. (54), is known to be given by [9]

$$
A_{a}^{(k)}=\sqrt{\frac{O_{M}^{(k)}}{d}}|a\rangle\langle a|+\sqrt{\frac{d-O_{M}^{(k)}}{d(d-1)}}(\mathbb{1}-|a\rangle\langle a|),
$$

where $\{|a\rangle\}_{a=1}^{d}$ is an arbitrary orthonormal basis. Thus, the largest $c$, given $F_{k}$ (i.e., given $O_{M}^{(k)}$ ), is

$$
c=\left[\sqrt{O_{M}^{(k)}}+\sqrt{(d-1)\left(d-O_{M}^{(k)}\right)}\right]^{2} .
$$

The corresponding POVM reads

$$
M_{a}^{(k)}=A_{a}^{(k) \dagger} A_{a}^{(k)}=\frac{O_{M}^{(k)}-1}{d-1}|a\rangle\langle a|+\frac{d-O_{M}^{(k)}}{d(d-1)} \mathbb{1},
$$

i.e., for this particular POVM, the optimal instrument, in terms of Kraus operators, is given by the Hermitian square root $A_{a}^{(k)}=\sqrt{M_{a}^{(k)}}$. One could continue the analysis using the (unaveraged) $d$-outcome measurement, Eq. (55), optimal for any achievable value of $F_{k}$. However, we will proceed in terms of the effective averaged covariant apparatuses with measurement outcomes given by the possible guesses. Covariant apparatuses may easily be constructed-this is a much harder task in the case of discrete apparatuses for encodings into higher-dimensional Hilbert spaces (cf. Ref. [20] for $N$ copies of a qubit).

It follows from Eq. (46) that any $\mathrm{SU}(d)$-covariant POVM on a qudit (with outcomes corresponding to guesses) has the operator density of the form

$$
\tilde{\mathcal{M}}^{\left(\varepsilon_{k}\right)}\left(\psi_{k}\right)=\left(1-\varepsilon_{k}\right) \mathbb{1}+\varepsilon_{k} \tilde{\mathcal{M}}\left(\psi_{k}\right),
$$

where $\tilde{\mathcal{M}}$ is the operator density of the optimal covariant POVM of the greedy-observers scenario, Eq. (35), and $\varepsilon_{k}$ parametrizes the strength of the measurement.

Then, Eq. (58) leads to

$$
O_{\mathcal{M}^{\left(\varepsilon_{k}\right)}}^{(k)}=1+\varepsilon_{k}(d-1),
$$

where we have used Eq. (52) in the form $O_{\mathcal{M}^{\left(\varepsilon_{k}\right)}}^{(k)}=$ $\int d \psi_{k} \operatorname{Tr}\left[\psi_{k} \tilde{\mathcal{M}}^{\left(\varepsilon_{k}\right)}\left(\psi_{k}\right)\right]$. Note that $1 \leqslant O_{\mathcal{M}^{\left(\varepsilon_{k}\right)}}^{(k)} \leqslant d$, depending on the greediness, or strength $\varepsilon_{k}, 0 \leqslant \varepsilon_{k} \leqslant 1$, of the $k$ th observer's measurement. Constructing the corresponding Hermitian-square-root Kraus operators $\mathcal{A}^{\left(\varepsilon_{k}\right)}$ defined by

$$
\tilde{\mathcal{A}}^{\left(\varepsilon_{k}\right)}(\psi)=\sqrt{O_{\mathcal{M}^{\left(\varepsilon_{k}\right)}}^{(k)}}|\psi\rangle\langle\psi|+\sqrt{\frac{d-O_{\mathcal{M}^{\left(\varepsilon_{k}\right)}}^{(k)}}{(d-1)}}(\mathbb{1}-|\psi\rangle\langle\psi|)
$$

( $\tilde{\mathcal{A}}$ is the operator density of the Kraus operator ${ }^{4} \mathcal{A}$, i.e., $\left.\mathcal{A}(\sqrt{d \psi})=\sqrt{d \psi} \tilde{\mathcal{A}}_{\psi}\right)$, we may verify that, for a given value of $F_{k}$, it induces the same channel as the minimal optimal measurement, Eq. (55). Thus, the Hermitian-square-root realization of the general weak covariant POVM, Eq. (58), gives the optimal covariant instrument.

Using Eqs. (59) and (50), we have

$$
F_{k}=\frac{1}{d}+\frac{\left(d O_{S}^{(k-1)}-1\right) \varepsilon_{k}}{d(d+1)}
$$

and from Eq. (48), with a pure initial state, Eq. (51) reads

$$
O_{S}^{(k)}=\frac{1}{d}+\frac{d-1}{d} \prod_{\beta=1}^{k} r_{\beta}
$$

Substituting the above equation into Eq. (61), we finally obtain

$$
F_{k}=\frac{1}{d}+\frac{\varepsilon_{k}(d-1)}{d(d+1)} \prod_{\beta=1}^{k-1} r_{\beta},
$$

where the $r_{\beta}$ can be expressed as a function of the parameter $\varepsilon_{\beta}$ by making use of Eqs. (54), (56), and (59),

$$
r_{\beta}=\frac{d-1+(2-d) \varepsilon_{\beta}+2 \sqrt{1+\varepsilon_{\beta}(d-1)} \sqrt{1-\varepsilon_{\beta}}}{d+1} .
$$

The above equation applies to general consecutive measurements on a $d$-dimensional system (single copy). Naturally, here, we recover the results of the greedy scenario, Eq. (40), with $N=1$, in the limit of most-informative measurements $\left(\varepsilon_{k}=1\right)$.

We next discuss the weak measurement case for $N$ copies of a qubit state.

\footnotetext{
${ }^{4}$ Here, the square root of a measure is only a formal notation. In expressions where probabilities and postmeasurement states are calculated, the measure always appears to the first power. A rigorous treatment of Radon-Nikodym derivatives of quantum instruments can be found in Refs. [23] and [24].
} 


\section{B. $N$ copies of a qubit}

Here, we consider a signal that is a state of $N$ copies of a two-dimensional unknown pure state. Again, we will assume that the observers measurements are restricted to the completely symmetric Hilbert space. Hence, this situation can be mapped to the problem of estimating the state of a single $D$-dimensional system $\left(D=d_{N}^{\text {sym }}=N+1\right)$, which is, however, known to belong to the restricted set of states from the orbit of a reference pure $N$-copy state generated by elements of the range of the symmetric representation of $\mathrm{SU}(2)$.

It turns out to be very useful for our purposes to recall an old result by Holevo [17]. He solved the unconstrained, or in our terminology, greedy optimization problem for generic mixed states $\varrho(\psi)$ drawn from a covariant family of states,

$$
\left\{\varrho(\psi)=U(g) \varrho\left(\psi_{\mathrm{ref}}\right) U(g)^{\dagger}, \quad U(g)=g^{\otimes N}, \quad g \in \mathrm{SU}(2)\right\},
$$

where $\left[g, \psi_{\text {ref }}\right]=0 \Rightarrow\left[g^{\otimes N}, \varrho\left(\psi_{\text {ref }}\right)\right]=0, \forall g \in \mathrm{SU}(2)$.

He found that the optimal fidelity $F(\varrho)$ is given by

$$
F=\frac{1}{2}\left(1+\frac{2\left\langle J_{\boldsymbol{n}\left(\psi_{\mathrm{ref}}\right)}\right\rangle_{\varrho\left(\psi_{\mathrm{ref}}\right)}}{N+2}\right)
$$

where

$$
\left\langle J_{\boldsymbol{n}(\psi)}\right\rangle_{\varrho(\psi)}:=\operatorname{Tr}\left[J_{\boldsymbol{n}(\psi)} \varrho(\psi)\right],
$$

and $J_{\boldsymbol{n}(\psi)}$ is the angular-momentum component in the direction fixed by the Bloch vector $\boldsymbol{n}(\psi)$. The optimal greedy covariant POVM is also proven to be given by $\tilde{\mathcal{M}}(\psi)=d_{N}^{\text {sym }}|\psi\rangle\left\langle\left.\psi\right|^{\otimes N}\right.$, independent of a particular family, Eq. (65).

The most general covariant POVMs one should consider here are ones that have a seed that commutes with $J_{\boldsymbol{n}\left(\psi_{\text {ref }}\right)}$ [17], i.e., the seed is diagonal in the $\{|j m\rangle\}$ basis [where we have chosen $\boldsymbol{n}\left(\psi_{\text {ref }}\right)$ as our quantization axis]. Note that, in principle, several weakness parameters could be included in the optimization, in contrast to the single parameter required in the previous subsection. Here we will make two simplifying assumptions - the assumption of considering single-parameter families of POVMs and the assumption of Hermitian-squareroot Kraus operators. That is, the measurement is given by a covariant POVM that, as before, is a convex combination of the optimal greedy POVM and the full identity, i.e.,

$$
\tilde{\mathcal{M}}^{(k), \varepsilon_{k}}(\psi)=\left(1-\varepsilon_{k}\right) \mathbb{1}+\varepsilon_{k} \tilde{\mathcal{M}}^{(k)}(\psi),
$$

where $\tilde{\mathcal{M}}^{(k)}$ is the operator density of the covariant greedy POVM optimal for the family of states at the input of $k$ th observer's apparatus, and $\varepsilon_{k}$ parametrizes the strength of the measurement. The corresponding Hermitian-square-root Kraus operators densities read,

$$
\tilde{\mathcal{A}}^{(k), \varepsilon_{k}}(\psi)=b_{k} \frac{\tilde{\mathcal{M}}^{(k)}(\psi)}{d_{N}^{\text {sym }}}+a_{k} \mathbb{1},
$$

where

$$
\begin{gathered}
a_{k}=\sqrt{1-\varepsilon_{k}}, \\
b_{k}=\sqrt{1+\left(d_{N}^{\text {sym }}-1\right) \varepsilon_{k}}-a_{k} .
\end{gathered}
$$

In Appendix B, it is shown that such evolution leads to a channel that leaves the postmeasurement state, after averaging over guesses, diagonal in the $\{|j m\rangle\}$ basis, and hence, it is of the form, Eq. (65). It follows that for each $k, \tilde{\mathcal{M}}^{(k)}=\tilde{\mathcal{M}}$, where $\tilde{\mathcal{M}}$ is given by Eq. (35). We emphasize that, although the above two restrictions seem to be a reasonable guess for a generalization of the optimal apparatus from the single-copy case, we do not have a proof that, for $N>1$, such apparatuses really are among the optimal ones. Therefore, it is only guaranteed that we obtain a lower bound $\mathbb{F}_{\text {eq }}$ on the maximum $\mathcal{F}_{\text {eq. }}$.

We start by rewriting the average fidelity $F_{1}^{\varepsilon_{k}}$ of a single estimation using the $\left(\varepsilon_{k}\right.$-strong) apparatus, Eq. (68), of the $k$ th observer's measuring on an arbitrary set of states in terms of estimation fidelity $F_{1}$, obtained using the apparatus of the greedy-observers problem,

$$
F_{1}^{\varepsilon_{k}}=\frac{\left(1-\varepsilon_{k}\right)}{2}+\varepsilon_{k} F_{1} .
$$

Then, by Eq. (66), for an arbitrary family of states, Eq. (65),

$$
F_{1}^{\varepsilon_{k}}=\frac{1}{2}\left(1+\varepsilon_{k} \frac{2\left\langle J_{\boldsymbol{n}(\psi)}\right\rangle_{\varrho(\psi)}}{N+2}\right) .
$$

Appendix B gives us the postmeasurement states for each step in a sequence of weak measurements given by Eq. (69) and thus, for each $k$, we can evaluate the average fidelity of the $k$ th observer $F_{k}^{\varepsilon}$ where $\varepsilon=\left(\varepsilon_{k}, \ldots, \varepsilon_{1}\right)$. Formally, this is performed according to Eq. (73) with $\varrho(\psi) \mapsto \hat{\rho}_{k-1}^{\varepsilon}=\chi^{\varepsilon_{k-1}} \circ$ $\cdots \circ \chi^{\varepsilon_{1}}(\varrho(\psi))$, i.e.,

$$
F_{k}^{\varepsilon}=F_{1}^{\varepsilon_{k}}\left(\hat{\rho}_{k-1}^{\varepsilon}\right)=\frac{1}{2}\left(1+\varepsilon_{k} \frac{2\left\langle J_{n}\right\rangle_{\hat{\rho}_{k-1}^{\varepsilon}}}{N+2}\right),
$$

where the following relation holds between the consecutive output states (see Appendix B),

$$
\left\langle J_{z}\right\rangle_{k+1}=\left(a_{k+1}^{2}+\frac{2 a_{k+1} b_{k+1}}{2 j+1}+\frac{b_{k+1}^{2} j}{(j+1)(2 j+1)}\right)\left\langle J_{z}\right\rangle_{k} .
$$

We are now in position to calculate two interesting scenarios where weak measurements need to be considered.

\section{EGALITARIAN STRATEGY}

We devise a protocol such that the estimation fidelity obtained by each observer is the same and maximal, i.e., we want to find the maximum fidelity $F_{k} \equiv \mathcal{F}_{\text {eq }}$ under the egalitarian constraints $F_{k}=F_{1}, \forall k \in\{2, \ldots, K\}$. The overall number of observers $K$ is fixed beforehand, and each observer knows his or her own tally number $k$ in the sequence. One can visualize this scenario as different apparatuses being delivered to the observers by an external party, or as observers sharing a single apparatus that adjusts its measurement-strength automatically before each measurement. We again require that each observer orients his or her apparatus independently and we do not allow communication between observers.

With these conditions, it is clear that the last observer will perform an optimal greedy measurement for the ensemble of states on the input of the apparatus, while going backward, each of the predecessor's measurements will be weaker and weaker, i.e., less and less demolishing.

With these considerations in mind, we can give the results for the two types of encodings discussed in Secs. V A and V B. 


\section{A. System of arbitrary dimension (single copy)}

Using Eq. (63), the condition $F_{k}=F_{k-1}$ translates into

$$
\varepsilon_{k-1}=\varepsilon_{k} r_{k-1},
$$

or, more explicitly through Eq. (64),

$$
\varepsilon_{k+1}=\frac{\varepsilon_{k}(d+1)}{d-1+(2-d) \varepsilon_{k}+2 \sqrt{1+\varepsilon_{k}(d-1)} \sqrt{1-\varepsilon_{k}}},
$$

where the initial condition $\varepsilon_{K}=1$ follows from the fact that, as mentioned above, the last, $K$ th, observer can measure greedily as there is no subsequent observer to care about. The recursion relations, Eq. (77), are quadratic and, hence, can be inverted analytically, providing all the measurement strengths $\varepsilon_{k}$ starting from $\varepsilon_{K}=1$. However, a closed-form solution for $\varepsilon_{1}$ seems to be hard to obtain for finite $K$. Nevertheless, for large $K$, we can obtain an asymptotic analytical expression of the fidelity and the initial $\varepsilon_{k}$ 's.

If $K \gg 1$, we expect the first measurements to be very weak, i.e., with $\varepsilon_{k} \ll 1$. Performing a Taylor expansion around $\varepsilon_{k}=0$ in the recursion relation, Eq. (77), we obtain an approximated relation for small values of $k$,

$$
\varepsilon_{k+1}=\varepsilon_{k}+\frac{d^{2}}{4(d+1)} \varepsilon_{k}^{3}
$$

or, by defining $\alpha(j)=\varepsilon_{K+1-j}$,

$$
\alpha(j)=\alpha(j+1)+\frac{d^{2}}{4(d+1)} \alpha(j+1)^{3},
$$

which holds for large values of $j$. In this regime, $\alpha(j)$ is vanishing small, and the difference equation can be written as an ordinary differential equation,

$$
\frac{d \alpha(x)}{\mathrm{d} x}=-\frac{d^{2}}{4(d+1)} \alpha(x)^{3},
$$

which yields

$$
\begin{aligned}
\alpha(j) & =\sqrt{\frac{2}{\left(j-j_{0}\right) d^{2} /(d+1)+2 \alpha\left(j_{0}\right)^{-2}}}, \\
& \simeq \sqrt{\frac{2(d+1)}{j d^{2}}},
\end{aligned}
$$

where $j_{0}$ is a fixed $\left(j_{0} \ll K\right)$ lower boundary of integration chosen such that the above approximations are valid. With this, we finally arrive at

$$
\varepsilon_{1}=\alpha(K) \simeq \frac{1}{d} \sqrt{\frac{2(d+1)}{K}} \quad(K \gg 1) .
$$

Inserting the above into $F_{1}$ of Eq. (63), we have, for large $K$, the maximal average fidelity of each egalitarian observer,

$$
\mathcal{F}_{\text {eq }}(K, d) \simeq \frac{1}{d}\left[1+\frac{d-1}{d} \sqrt{\frac{2}{(d+1) K}}\right] .
$$

Let us note that a related problem of informationdisturbance trade-off in sequential weak measurements on a qudit signal has been studied in Ref. [25]. There, users are not considered fully independent, in particular, they share a reference frame, which allows them to obtain an estimation fidelity that does not decrease with the number of users.

\section{B. $N$ copies of a qubit}

From Eq. (74), it follows that, in order for every observer to have the same fidelity $\left(F_{k}^{\varepsilon}=F_{l}^{\varepsilon}, \forall l, k\right.$ subject to $0<l<$ $k \leqslant K)$, it must hold that

$$
\varepsilon_{k}\left\langle J_{n}\right\rangle_{\hat{\rho}_{k-1}^{\varepsilon}}=\varepsilon_{l}\left\langle J_{\boldsymbol{n}}\right\rangle_{\hat{\rho}_{l-1}^{\varepsilon}} .
$$

To proceed further, we need to evaluate how the channels $\chi^{\varepsilon_{k}}$ transform $\left\langle J_{\boldsymbol{n}}\right\rangle$ for the relevant states, which we do in Appendix B. Comparing Eq. (85), with $l=k+1$, to Eq. (B11) of Appendix B, we get a recursion relation for the strength parameters $\varepsilon_{k}$,

$$
\varepsilon_{k+1}=\frac{(N+1)(N+2) \varepsilon_{k}}{(N+1)^{2}+(N-1)\left(1-2 \varepsilon_{k}\right)+4 \sqrt{\left(1-\varepsilon_{k}\right)\left(1+N \varepsilon_{k}\right)}-2},
$$

where $\varepsilon_{K}=1$. Again, this recursion relation gives all the strength parameters $\varepsilon_{k}$ starting on reverse from $k=K$. To obtain the fidelity, one needs to solve the recurrence relation, Eq. (86), for $k=1$, and then, use Eq. (74) to get

$$
\mathbb{F}_{\mathrm{eq}}(N, K)=\frac{1}{2}\left(1+\Delta_{\mathrm{eq}}\right)
$$

where

$$
\Delta_{\text {eq }}=\varepsilon_{1}(K, N) \frac{N}{N+2} .
$$

The presence of the square roots in Eq. (86) prevents the existence of a closed expression for $\varepsilon_{1}$. However, in the asymptotic regimes of large $K$ or large $N$, we can find the leading order behaviors of $\varepsilon_{1}$ and $\mathbb{F}_{\mathrm{eq}}$.
Let us first consider the situation $K \gg N$. As above, we expect the first measurements to be very weak, i.e., with $\varepsilon_{k} \ll$ $1, k \leqslant k_{0} \ll K$. Thus, we can perform a Taylor expansion in $\varepsilon$ around the point $\varepsilon=0$ in Eq. (86) and get the approximate relation,

$$
\varepsilon_{k+1}=\varepsilon_{k}+\frac{N+1}{2(N+2)} \varepsilon_{k}^{3}
$$

which, proceeding as in Eqs. (78)-(83), leads to

$$
\varepsilon_{1} \simeq \sqrt{\frac{N+2}{(N+1) K}} \quad(K \gg N)
$$


Inserting Eq. (90) into Eq. (88), we have

$$
\Delta_{\mathrm{eq}} \simeq \frac{N}{\sqrt{(N+1)(N+2) K}} \quad(K \gg N) .
$$

Again, we obtain a behavior for a large number of observers as $\Delta \sim 1 / \sqrt{K}$. This result deserves some comments, since one would naively expect that $\Delta$ degrades with the inverse of the number of observers, i.e., as $\Delta \sim 1 / K$. The realization of the POVM, Eq. (68), as an instrument given by Hermitiansquare-root Kraus operators, Eq. (69), is crucial to obtain this square-root degradation. Had we used a more destructive realization, we would indeed have obtained $\Delta \sim 1 / K$. For instance, if we realize the POVM, Eq. (68), as a stochastic measurement such that with probability $\left(1-\varepsilon_{k}\right)$, the outcome is just guessed, i.e., nothing is done to the state, and with probability $\varepsilon_{k}$, the optimal greedy covariant measurement is performed, the (relevant part of the) channel induced by such measurements is $\chi_{\varepsilon_{k}}^{\prime}=\left(1-\varepsilon_{k}\right) \mathrm{Id}+\varepsilon_{k} \chi$, where Id is the identity channel and $\chi$ is the channel induced by the optimal greedy measurements. In this case,

$$
\left\langle J_{n}\right\rangle_{k+1}=\left(1-\frac{\varepsilon_{k+1}}{N / 2+1}\right)\left\langle J_{n}\right\rangle_{k} .
$$

The condition, Eq. (85), then leads to the recurrence relation that can easily be solved and gives

$$
\varepsilon_{k}=\frac{N / 2+1}{N / 2+K-k+1} \rightarrow \varepsilon_{1}=\frac{N+2}{N+2 K},
$$

which yields

$$
\Delta=\frac{N}{N+2 K}
$$

and, clearly, for $K \gg N, \Delta \rightarrow N /(2 K)$. Note, in addition, that Eq. (94) is precisely the result that one obtains with a strategy where each observer performs a greedy measurement on a fraction $\tilde{N}=N / K$ of the copies. Indeed, from Eq. (39) with $d=2$, one has $\Delta=\tilde{N} /(\tilde{N}+2)=N /(N+2 K)$.

Now, we proceed to study the case where the number of copies is asymptotically large, i.e., $N \gg 1$. The large- $N$ expansion in Eq. (86) yields

$$
\varepsilon_{k+1}=\varepsilon_{k}+\frac{2 \varepsilon_{k}^{2}}{N}
$$

which, starting from $\varepsilon_{K}=1$, gives at the order $1 / N$,

$$
\varepsilon_{1}=1-\frac{2(K-1)}{N} \text {. }
$$

Hence, from Eq. (88), we have for $N \gg K$,

$$
\begin{aligned}
\Delta_{\mathrm{eq}} & \simeq 1-\frac{2 K}{N+2}, \\
& \simeq 1-\frac{2 K}{N} .
\end{aligned}
$$

Naturally, for $K=1$ in Eq. (97), we recover the well-known result of the optimal measurement of $N$ copies of a qubit $[10,26]$ given by Eq. (40), $d=2, k=1$. The efficiency of this egalitarian strategy in the $N \gg K$ regime coincides with the

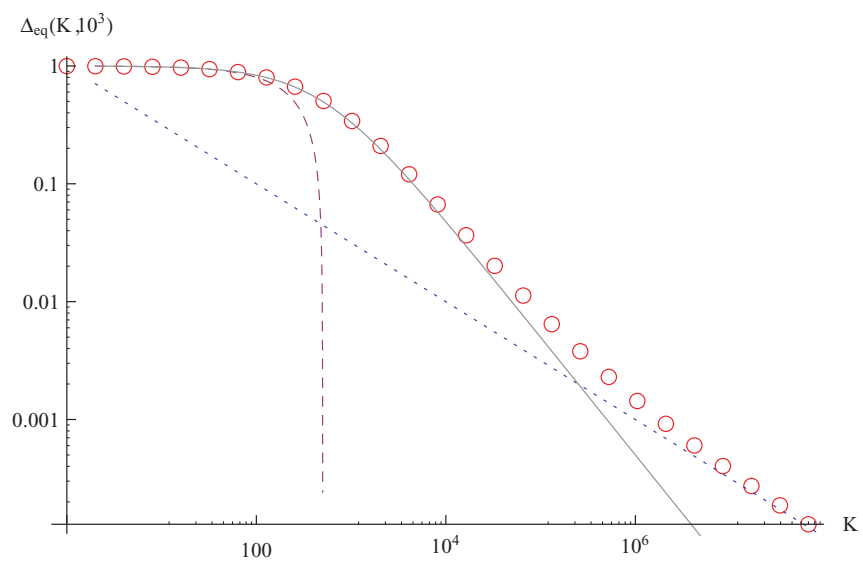

FIG. 1. (Color online) The observers' measurement performance $\Delta_{\text {eq }}$ as a function of the number of observers $K$. The solution given by the exact recurrence relations [Eq. (86)] (circles). The approximate solution for $K \gg N$ [Eq. (91)] (dotted) and $N \gg K$ [Eq. (97)] (dashed). Stochastic strategy [Eq. (94)] (solid). The solid line also depicts the performance of each observer measuring only a fraction $\tilde{N}=N / K$ of copies.

stochastic strategy, Eq. (94), and the greedy one where each observer measures only the fraction $\tilde{N}=N / K$ of the copies.

In Fig. 1, we plot the observers' performance $\Delta_{\text {eq }}$ obtained by a numerical evaluation of the exact recurrence relation, Eq. (86), as well as the approximations of the limiting regimes discussed above. $N=10^{3}$ has been chosen to accommodate all the regimes. The stochastic strategy performance, [Eq. (94)], which coincides with the greedy one over $N / K$ copies, is also plotted for reference. Notice that it gives a very good approximation for the true values of the fidelity even for $K \gtrsim N$. The deviation starts to be appreciable only beyond $K \simeq 10^{4}$.

\section{PRIVILEGED OBSERVER STRATEGY}

Here, we consider a scenario where all observers use exactly the same measurement device (up to the unknown relative orientation), but this is provided, or is tailored, by a particular, say the $K$ th, observer who wants to optimize his or her own estimation fidelity. That is, the right compromise has to be found, i.e., the optimal measurement strength $\varepsilon$, between these two extreme cases: (i) Choose a very weak measurement that prevents the $(K-1)$ previous observers to extract much information from the state and, thus, to facilitate little disturbance, but at the same time, prevents gaining information about it when the observer's turn comes; (ii) choose the most informative measurement that will guarantee that the maximum information is extracted from the state the privileged observer receives but, by then, all the previous most informative measurements will have significantly ruined the input state.

\section{A. Single qudit}

For one copy, covariant POVMs with the Hermitian-squareroot update rule are optimal. They are of the form of the one-parameter family given by Eq. (58). Based on Eq. (63), the fidelity $F_{K}=\left[1+(d-1) \Delta_{K}\right] / d$ of the last observer is 
determined by

$$
\Delta_{K}=\frac{\varepsilon}{d+1} r^{K-1}
$$

where $r$ is defined in Eq. (64).

We can obtain analytical results in the asymptotic regime $K \gg 1$. Here, we again have $\varepsilon \ll 1$, and Taylor expanding $\Delta_{K}$ of Eq. (99) around $\varepsilon=0$, we have

$$
\begin{aligned}
\Delta_{K} & =\frac{\varepsilon}{d+1}\left(1-\frac{d^{2} \varepsilon^{2}}{4(d+1)}\right)^{K-1} \\
& \simeq \frac{\varepsilon}{d+1} \exp \left[-\frac{d^{2} K}{4(d+1)} \varepsilon^{2}\right]
\end{aligned}
$$

The value of $\varepsilon$ that maximizes the above expression is

$$
\varepsilon^{*}=\sqrt{\frac{2(d+1)}{d^{2} K}},
$$

which inserted in Eq. (101) yields

$$
\Delta_{K, \max } \simeq \sqrt{\frac{2}{e(d+1) d^{2} K}} \quad(K \gg 1) .
$$

Observe that it exhibits the same characteristic square-root decay $1 / \sqrt{K}$ as in the egalitarian case.

\section{B. $N$ copies of a qubit}

As in the egalitarian case, we restrict our attention to weak measurements of the type of Eq. (68) and the Hermitiansquare-root update rule. From the results of previous sections, the computation of the fidelity and measurement strength are quite straightforward. Based on Eq. (75), the fidelity $F_{K}=$ $\left(1+\Delta_{K}\right) / 2$ of the privileged observer $K$ is determined by

$$
\begin{aligned}
\Delta_{K} & =\varepsilon \frac{N / 2}{N / 2+1}\left\langle J_{n}\right\rangle_{\rho_{k-1}}, \\
& =\varepsilon \frac{N}{N+2}\left(\frac{A(\varepsilon)}{N+1}\right)^{K-1},
\end{aligned}
$$

where

$$
A(\varepsilon)=2 a b+(N+1) a^{2}+\frac{N}{N+2} b^{2},
$$

with $a$ and $b$, now independent of $k$, defined as in Eqs. (70) and (71).

Let us obtain analytical expressions of the fidelity in the asymptotic regimes. If $K \gg N$, we expect $\varepsilon \ll 1$ and Taylor expanding $\Delta_{K}$ around $\varepsilon=0$, and taking two lowest orders in $\varepsilon$, we get

$$
\begin{aligned}
\Delta_{K} & \simeq \frac{\varepsilon}{N+2}\left(1-\frac{(N+1) \varepsilon^{2}}{2(N+2)}\right)^{K-1} \\
& \simeq \frac{\varepsilon}{N+2} \exp \left[-\frac{(N+1) K}{2(N+2)} \varepsilon^{2}\right]
\end{aligned}
$$

Proceeding as in the previous subsection, the optimal value of $\Delta_{K}$ reads

$$
\Delta_{K, \max } \simeq \sqrt{\frac{N^{2}}{e(N+1)(N+2) K}} .
$$

Again, the fidelity degrades as $1 / \sqrt{K}$ instead of the naive $1 / K$ behavior.
In the other regime $N \gg K$, we expect $\varepsilon \rightarrow 1$. Then, we Taylor expand Eq. (105) in the variable $(1-\varepsilon)$ around 0 and take terms up to the first power of $(1-\varepsilon)$. Maximization of $\Delta_{K}$ gives the optimal $\varepsilon$, which, up to the first nonvanishing order, reads

$$
\varepsilon^{*}=1-\frac{4(K-1)^{2}}{N^{3}} .
$$

This value can be taken to be $\varepsilon^{*}=1$, as the corrections will not affect the $1 / N$ term of the fidelity. Therefore, we should obtain the same results for the fidelity of a greedy scenario with an asymptotically large number of copies as discussed at the end of Sec. IV. Indeed, the expansion of $\Delta_{K}$ for $N \rightarrow \infty$ at the first order does not include any $(1-\varepsilon)$ terms, and we obtain

$$
\Delta_{K, \max }=1-\frac{2 K}{N},
$$

or, equivalently,

$$
F_{K} \simeq 1-\frac{K}{N}
$$

Actually, we notice that, in this regime, at first order in $1 / N$, a greedy strategy of each observer in $N / K$ copies, the egalitarian and the privileged-man scenario yield the same accuracy.

\section{CONCLUSIONS}

We have investigated to what extent can a series of independent observers estimate an unknown state of a $d$-dimensional system by performing consecutive measurements over the very same system. More generally, we have studied the case where $N$ copies of an unknown state are given and when more general encodings into a signal system with a larger Hilbert space are permitted. This has allowed us to assess how large does the signal system need to be so that a given number of observers can obtain reasonable estimates, i.e., to behave classically with regard to the readout of the state encoded in the system. We obtain that, with the optimal encoding, the size has to be, at least, $N \sim \sqrt{K}$. This is a quadratic improvement over the case of a signal consisting of copies of the encoded state for which the size must be, at least, $N \sim K$.

In addition, we have studied more general ways to distribute the (limited) information on the unknown quantum state among different observers, still under the constraint that they measure one right after the other. We have studied a strategy that leads to equal fidelities for all observers (egalitarian strategy) and a second strategy where all observers are constrained to use the same apparatus, and the goal is to maximize the estimation fidelity of a privileged observer, which is at the $K$ th position in the measurement queue. In both scenarios, weak measurements are required. Since the systems are measured several times with observers trying to scavenge the information contained in them after each measurement, the choice of the Kraus operators, i.e., the choice of the instrument implementing a given measurement and the trade-off between fidelity and disturbance $[9,27,28]$ play a crucial role. We have seen that, for instance, the update rule given by Hermitian-square-root Kraus operators yields, in the asymptotic regime of a large number of systems, a fidelity that degrades as the square root of the number of observers, in 
contrast to a linear degradation given by a stochastic realization of the same POVM.

Our results can also shed some light on how quantum reference frames degrade with use. This problem was first addressed in Refs. [22,29] (see also Ref. [30]). There, the authors consider a setting where one has a quantum directional reference and a set of reservoir spin particles that are pure and are oriented randomly with respect to the reference. One of the goals is to correctly identify the mutual alignment or antialignment of the reference and the spin particle by performing a suitable (in general, collective) measurement on the two systems. As the number of spins measured grows, the success probability of correct identification of the orientation drops. The rate of decrease quantifies the degradation of the directional reference with the number of measurements performed. Our results can be extended to address separable versions of this problem, that is, settings where, first, one measures the quantum reference and then one performs reference-dependent tasks, such as, e.g., the one just described. These problems are under current investigation, and some further details can be found in the dissertation [31].

\section{ACKNOWLEDGMENTS}

This work was supported by the European Union Projects No. Q-essence, No. HIP 221889, by the Projects No. CE SAV, No. QUTE, No. meta-QUTE-IMTS NFP26240120022, No. APVV-0673-07, and No. VEGA 2/0092/09, by the Spanish MEC Contracts No. FIS2008-01236 (E.B.), No. PR20100367, and No. QOIT Consolider-Ingenio 2006-00019, and by the Catalan government, Grant No. CIRIT 2009GR-0985.

\section{APPENDIX A: EVALUATION OF THE INTEGRAL, EQ. (18)}

Choosing, for the sake of calculations, an arbitrary pure reference state $\psi_{0} \in \mathcal{S}\left(\mathcal{H}_{d}\right)$, we can parametrize the pure states by elements $g \in \mathrm{SU}(d)$ and can replace the integration over the pure states by integration over the group $\mathrm{SU}(d)$. The integral, Eq. (18), becomes

$$
\int_{g \in \mathrm{SU}(d)} d \mu(g) \boldsymbol{n}(g) \tilde{p}(\hat{g} \mid g),
$$

where $\boldsymbol{n}(g)$ is a $d$-dimensional Bloch vector parametrizing the state $|\psi(g)\rangle\langle\psi(g)|$ and

$$
\tilde{p}(\hat{g} \mid g)=\operatorname{Tr}[\tilde{\mathcal{M}}(\hat{g}) \rho(g)],
$$

where $\mathcal{S}\left(\mathcal{H}_{D}\right) \ni \rho(g)=\mathcal{U}(g) \rho_{0} \mathcal{U}(g)^{\dagger}$. Note that, due to the covariance of both the measurement and the states $\rho(g)$, it holds that

$$
\operatorname{Tr}[\tilde{\mathcal{M}}(\bar{g} \hat{g}) \rho(\bar{g} g)]=\operatorname{Tr}\left[\mathcal{U}^{\prime}(\bar{g}) \tilde{\mathcal{M}}(\hat{g}) \mathcal{U}^{\prime}(\bar{g})^{\dagger} \mathcal{U}(\bar{g}) \rho(g) \mathcal{U}(\bar{g})^{\dagger}\right]
$$

For optimal covariant encoding-decoding schemes, it holds that the representations are the same, i.e., $\mathcal{U}^{\prime}(g)=\mathcal{U}(g)$, hence,

$$
\tilde{p}(\bar{g} \hat{g} \mid \bar{g} g)=\tilde{p}(\hat{g} \mid g) \text {. }
$$

A $d$-dimensional system in a pure state $\psi=|\psi\rangle\langle\psi|$ can be parametrized as

$$
\psi=\frac{1}{d}\left\{\mathbb{1}+\kappa_{d} n^{a} T_{a}\right\}
$$

where

$$
\left\{T_{a}, T_{b}\right\}=\frac{\delta_{a b}}{d}+d_{a b}^{c} T_{c},
$$

with the generators defined as half the standard Gell-Mann matrices,

$$
\kappa_{d}=\sqrt{2 d(d-1)}
$$

and $n^{a}$ are the components of a $\left(d^{2}-1\right)$-dimensional unit vector: $\boldsymbol{n}=\left(n^{1}, n^{2}, \ldots, n^{d^{2}-1}\right)$, which we refer to as the Bloch vector. This follows from imposing on $\psi$ the conditions $\operatorname{Tr} \psi=$ 1 and $\operatorname{Tr} \psi^{2}=1$.

Not any unit vector $\boldsymbol{n}$ is allowed. By imposing the condition $\psi=\psi^{2}$, we get further constraints,

$$
n^{a}=\frac{\kappa_{d}}{2(d-2)} d_{b c}^{a} n^{b} n^{c} .
$$

Any pure state can be obtained by applying a $\mathrm{SU}(d)$ transformation to the reference state,

$$
\left|\psi_{0}\right\rangle=\left(\begin{array}{c}
0 \\
0 \\
\vdots \\
0 \\
1
\end{array}\right)
$$

Note that

$$
\psi_{0}=\left|\psi_{0}\right\rangle\left\langle\psi_{0}\right|=\frac{1}{d}\left\{\mathbb{1}-\kappa_{d} T_{d^{2}-1}\right\}
$$

since

$$
T_{d^{2}-1}=\frac{1}{\sqrt{2 d(d-1)}}\left(\begin{array}{ccccc}
1 & 0 & \cdots & 0 & 0 \\
0 & 1 & \cdots & 0 & 0 \\
\vdots & \vdots & \ddots & \vdots & \vdots \\
0 & 0 & \cdots & 1 & 0 \\
0 & 0 & \cdots & 0 & 1-d
\end{array}\right)
$$

(the normalization ensures that $\operatorname{Tr}\left[T_{d^{2}-1}^{2}\right]=1 / 2$ ). Hence, the reference Bloch vector is

$$
\boldsymbol{n}_{0}=(\underbrace{0,0, \ldots, 0}_{d^{2}-2},-1),
$$

i.e., its components are

$$
n_{0}^{d^{2}-1}=-1, \quad n_{0}^{a}=0, \quad \text { if } a \neq d^{2}-1 .
$$

Note that $\left|\psi_{0}\right\rangle$ is covariant under the $\mathrm{SU}(d-1) \subset \mathrm{SU}(d)$ transformation of the form

$$
\tilde{U} \equiv U(\tilde{g})=\left(\begin{array}{ccccc}
U_{11} & U_{12} & \cdots & U_{1 d-1} & 0 \\
U_{21} & U_{22} & \cdots & U_{2 d-1} & 0 \\
\vdots & \vdots & \ddots & \vdots & \vdots \\
U_{d-11} & U_{d-12} & \cdots & U_{d-1 d-1} & 0 \\
0 & 0 & \cdots & 0 & 1
\end{array}\right) .
$$

Hence,

$$
\begin{aligned}
\left\langle\psi_{0} \mid \psi(g)\right\rangle & \equiv\left\langle\psi_{0}|U(g)| \psi_{0}\right\rangle \\
& =\left\langle\psi_{0}|\tilde{U} U(g)| \psi_{0}\right\rangle=\left\langle\psi_{0} \mid \psi(\tilde{g} g)\right\rangle \\
& =\left\langle\psi_{0}|U(g) \tilde{U}| \psi_{0}\right\rangle=\left\langle\psi_{0} \mid \psi(g \tilde{g})\right\rangle .
\end{aligned}
$$


Moreover, due to the covariance of the encoding, it also has to hold that

$$
\operatorname{Tr}\left[\rho_{0} \rho(g)\right]=\operatorname{Tr}\left[\rho_{0} \rho(g \tilde{g})\right] .
$$

We use the group parameters $g$ to label the different states according to

$$
|\psi(g)\rangle\left\langle\psi(g)|=U(g)| \psi_{0}\right\rangle\left\langle\psi_{0}\right| U^{\dagger}(g) .
$$

It follows that

$$
n^{a}(g) T_{a}=U(g), \quad n_{0}^{a} T_{a} U^{\dagger}(g)=A_{a}^{b}(g) n_{0}^{a} T_{b},
$$

where $A_{b}^{a}(\bar{g})$ belongs to the adjoint representation and we have used that

$$
U(g) T_{a} U^{\dagger}(g)=A_{a}^{b}(g) T_{b}
$$

We see that

$$
n^{a}(g)=A_{b}^{a}(g) n_{0}^{b}
$$

In general,

$$
n^{a}(g)=A_{b}^{a}(g) A_{c}^{b}\left(\bar{g}^{-1}\right) n^{c}(\bar{g})=A_{c}^{a}\left(g \bar{g}^{-1}\right) n^{c}(\bar{g}),
$$

from which

$$
n^{a}(g \bar{g})=A_{b}^{a}\left(g \bar{g} \bar{g}^{-1}\right) n^{b}(\bar{g})=A_{b}^{a}(g) n^{b}(\bar{g}) .
$$

Let us now consider the integral,

$$
V^{a}(\hat{g}) \equiv \int \mu(g) n^{a}(g) \tilde{p}(\hat{g} \mid g)
$$

Here, $\tilde{p}(\hat{g} \mid g)$ is the conditional-probability density, Eq. (A2). Let $\bar{U}=U(\bar{g})$ be any $\mathrm{SU}(d)$ transformation. We have

$$
\begin{aligned}
V^{a}(\bar{g} \hat{g}) & =\int d \mu(g) n^{a}(g) \tilde{p}(\bar{g} \hat{g} \mid g) \\
& =\int d \mu\left(\bar{g}^{-1} g\right) n^{a}\left(\bar{g} \bar{g}^{-1} g\right) \tilde{p}\left(\bar{g} \hat{g} \mid \bar{g} \bar{g}^{-1} g\right) \\
& =A_{b}^{a}(\bar{g}) \int d \mu\left(\bar{g}^{-1} g\right) n^{a}\left(\bar{g}^{-1} g\right) \tilde{p}\left(\hat{g} \mid \bar{g}^{-1} g\right) \\
& =A_{b}^{a}(\bar{g}) V^{b}(\hat{g}),
\end{aligned}
$$

where we have used the invariance of the Haar measure $d \mu(g)$ and the invariance of the probability density, Eq. (A3).

We see that, in particular,

$$
V^{a}(\hat{g})=A_{b}^{a}(\hat{g}) V^{b}(\mathbf{0}),
$$

where $\mathbf{0}$ denotes the identity parameters. That is,

$$
V^{b}(\mathbf{0})=\int d \mu(g) n^{b}(g) \tilde{p}(\mathbf{0} \mid g) .
$$

We now wish to show that, as expected, $V^{b}(\mathbf{0}) \propto n_{0}^{b}$. We proceed as follows. From

$$
T_{b} V^{b}(\mathbf{0})=\int d \mu(g) T_{b} n^{b}(g) \tilde{p}(\mathbf{0} \mid g)
$$

we observe that

$$
\begin{aligned}
\tilde{U} T_{b} V^{b}(\mathbf{0}) \tilde{U}^{\dagger} & =\int d \mu(\tilde{g} g) T_{b} n^{b}(\tilde{g} g) \tilde{p}(\mathbf{0} \mid g) \\
& =\int d \mu(\tilde{g} g) T_{b} n^{b}(\tilde{g} g) \tilde{p}(\mathbf{0} \mid \tilde{g} g) \\
& =T_{b} V^{b}(\mathbf{0}),
\end{aligned}
$$

where we have used Eq. (A6) in the form $\tilde{p}(\mathbf{0} \mid g)=\tilde{p}(\mathbf{0} \mid \tilde{g} g)$. Hence, according to Schur's lemma, $T_{b} V^{b}(\mathbf{0})$ must be the identity in the subspace corresponding to $\mathrm{SU}(d-1)$, i.e., proportional to $T_{d^{2}-1}$, from where the desired result follows immediately. Note that, from this, it also follows that

$$
V^{a}(\hat{g}) \propto A_{b}^{a}(\hat{g}) n_{0}^{b}=n^{b}(\hat{g}),
$$

or, more explicitly,

$$
\int d \mu(g) \boldsymbol{n}(g) \tilde{p}(\hat{g} \mid g)=\Delta \boldsymbol{n}(\hat{g}),
$$

where $\Delta$ is a constant.

\section{APPENDIX B: THE CHANNEL INDUCED BY THE MEASUREMENTS, EQ. (69), ON $N$ COPIES OF A QUBIT}

First, we compute the action of the channel induced by the $\mathrm{SO}(3)$-covariant measurement of the greedy strategy over a generic state,

$$
\hat{\rho}=\sum_{m=-j}^{m=j} s_{m}|j m\rangle\langle j m|
$$

In this case, we recall that the optimal covariant measurement has the operator density,

$$
\tilde{\mathcal{M}}(\boldsymbol{n})=(2 j+1)|j j ; \boldsymbol{n}\rangle\langle j j ; \boldsymbol{n}|,
$$

where $|j j ; \boldsymbol{n}\rangle$ is the rotated state from the $z$ direction (defined by the diagonalization axis of $\hat{\rho}$ ) into the $\boldsymbol{n}$ direction. The channel action is given by

$$
\chi(\hat{\rho})=(2 j+1) \sum_{m} s_{m} \int d \boldsymbol{n}|\langle j m \mid j j ; \boldsymbol{n}\rangle|^{2}|j j ; \boldsymbol{n}\rangle\langle j j ; \boldsymbol{n}| .
$$

It is easy to see that this operator is invariant under rotations along the $z$ axis and, therefore, is diagonal in the $|j m\rangle$ basis,

$$
\chi(\hat{\rho})=\sum_{m^{\prime}} c_{m^{\prime}}\left|j m^{\prime}\right\rangle\left\langle j m^{\prime}\right| .
$$

We further notice that

$$
\int d \boldsymbol{n}|j j ; \boldsymbol{n}\rangle\langle j j ; \boldsymbol{n}|\otimes| j j ; \boldsymbol{n}\rangle\langle j j ; \boldsymbol{n}|=\frac{\mathbb{1}^{(2 j)}}{4 j+1},
$$

where $\mathbb{1}^{(2 j)}=\sum_{M}|2 j ; M\rangle\langle 2 j ; M|$ is the projector onto the symmetric space of dimension $4 j+1$. Hence, we have

$$
c_{m^{\prime}}=\sum_{m} \Lambda_{m^{\prime}}^{m} s_{m}
$$

with

$$
\begin{aligned}
& \Lambda_{m^{\prime}}^{m}=\frac{2 j+1}{4 j+1}\left|\left\langle j m, j m^{\prime} \mid 2 j \quad m+m^{\prime}\right\rangle\right|^{2} \\
& =\frac{2 j+1}{4 j+1}\left(\begin{array}{c}
2 j \\
j+m
\end{array}\right)\left(\begin{array}{c}
2 j \\
j+m^{\prime}
\end{array}\right)\left(\begin{array}{c}
4 j \\
2 j+m+m^{\prime}
\end{array}\right)^{-1},
\end{aligned}
$$

where $\left\langle j m, j m^{\prime} \mid 2 j m+m^{\prime}\right\rangle$ is the Clebsch-Gordan coefficient of the composition $\mathbf{j} \otimes \mathbf{j} \rightarrow \mathbf{2} \mathbf{j}$.

As shown in the main text, to compute the fidelity, it is sufficient to calculate the expectation value of the spin component $J_{z}$. If Eq. (B1) is the state after $k$ uses of the channel 
and $\chi(\hat{\rho})$ is the state after $k+1$ uses, it is straightforward to obtain

$$
\left\langle J_{z}\right\rangle_{k+1}=\sum_{m m^{\prime}} m^{\prime} \Lambda_{m^{\prime}}^{m} s_{m}=\sum_{m} \frac{j}{j+1} m s_{m}=\frac{j}{j+1}\left\langle J_{z}\right\rangle_{k} .
$$

We can now proceed to compute the action of the channel and the quantity determining the fidelities for the weak measurements considered in the main text. In this case, the covariant POVM elements are given by operator density,

$$
\widetilde{\mathcal{M}}(\boldsymbol{n})=(1-\varepsilon) \mathbb{1}+\varepsilon(2 j+1)|j j ; \boldsymbol{n}\rangle\langle j j ; \boldsymbol{n}|,
$$

where the parameter $\varepsilon$ quantifies the strength of the measurement. The corresponding Kraus-operator densities are $\widetilde{\mathcal{A}}(\boldsymbol{n})=\sqrt{\widetilde{\mathcal{M}}(\boldsymbol{n})}$, which explicitly read

$$
\widetilde{\mathcal{A}}(\boldsymbol{n})=a \mathbb{1}+b|j j ; \boldsymbol{n}\rangle\langle j j ; \boldsymbol{n}|,
$$

where $a=\sqrt{1-\varepsilon}$ and $b=\sqrt{1+2 j \varepsilon}-\sqrt{1-\varepsilon}$. The action of the channel is fully determined from

$$
\begin{aligned}
& \chi^{\varepsilon}(|j m\rangle\langle j m|) \\
&= a^{2}|j m\rangle\langle j m|+a b \int d \boldsymbol{n}\langle j m \mid j j, \boldsymbol{n}\rangle(|j m\rangle\langle j j ; \boldsymbol{n}| \\
&\quad+|j j ; \boldsymbol{n}\rangle\langle j m|)+b^{2} \int d \boldsymbol{n}|\langle j m \mid j j ; \boldsymbol{n}\rangle|^{2}|j j ; \boldsymbol{n}\rangle\langle j j ; \boldsymbol{n}| .
\end{aligned}
$$

Using the same techniques as in the previous case, we obtain

$$
\begin{aligned}
\chi^{\varepsilon}(\hat{\rho}) & \equiv \sum_{m m^{\prime}} s_{m} \tilde{\Lambda}_{m^{\prime}}^{m} s_{m} \\
& =\sum_{m} s_{m} \frac{a^{2}(2 j+1)+2 a b}{2 j+1}|j m\rangle\langle j m|+\frac{b^{2}}{2 j+1} \chi(\hat{\rho}),
\end{aligned}
$$

where $\chi(\hat{\rho})$ is the action of the greedy channel, Eq. (B3), and $\tilde{\Lambda}_{m^{\prime}}^{m}=\frac{a^{2}(2 j+1)+2 a b}{2 j+1} \delta_{m^{\prime}}^{m}+\frac{b^{2}}{2 j+1} \Lambda_{m^{\prime}}^{m}$.

We finally compute the relation of the expectation values of the operator $J_{z}$ before and after the use of the channel. The analog of Eq. (B7) now reads

$$
\begin{aligned}
\left\langle J_{z}\right\rangle_{k+1} & =\sum_{m m^{\prime}} m^{\prime} \tilde{\Lambda}_{m^{\prime}}^{m} s_{m} \\
& =\left(a_{k+1}^{2}+\frac{2 a_{k+1} b_{k+1}}{2 j+1}+\frac{b_{k+1}^{2} j}{(j+1)(2 j+1)}\right)\left\langle J_{z}\right\rangle_{k},
\end{aligned}
$$

where the label $k$ in the parameters $a_{k}$ and $b_{k}$ simply takes into account that the strength $\varepsilon_{k}$ can vary from one measurement to another.

\section{APPENDIX C: THE AVERAGE CHANNEL INDUCED BY SINGLE-KRAUS-OPERATOR MEASUREMENTS ON A SINGLE QUDIT}

We show that the optimal weak instrument, i.e., one maximizing the next observer's fidelity given the current observer's fidelity for a qudit, induces a channel that has the effect of adding a portion of total mixture to the encoding state. We first collect some mathematical results concerning unitary group integrals that will extensively be used below. For matrices $g$ belonging to the fundamental representation of $\mathrm{SU}(d)$ and denoting by $d \mu(g)$, the corresponding Haar measure, we have

$$
\int d \mu(g) g_{i}^{j} g_{r}^{\dagger s}=\frac{\delta_{i}^{s} \delta_{r}^{j}}{d},
$$

and, similarly,

$$
\begin{aligned}
\int d \mu(g) g_{i}^{j} g_{k}^{l} g_{r}^{\dagger s} g_{t}^{\dagger v}= & \frac{\left(\delta_{i}^{s} \delta_{k}^{v}+\delta_{i}^{v} \delta_{k}^{s}\right)\left(\delta_{r}^{j} \delta_{t}^{l}+\delta_{r}^{l} \delta_{t}^{j}\right)}{2 d(d+1)} \\
& +\frac{\left(\delta_{i}^{s} \delta_{k}^{v}-\delta_{i}^{v} \delta_{k}^{s}\right)\left(\delta_{r}^{j} \delta_{t}^{l}-\delta_{r}^{l} \delta_{t}^{j}\right)}{2 d(d-1)} .
\end{aligned}
$$

The last result can most easily be seen by writing the integral above as

$$
\int d \mu(g)(\square \square \oplus \square) \otimes(\square \square \oplus \square)^{\dagger},
$$

and recalling the orthogonality relations of the irreducible representations of unitary groups, which state that

$$
\begin{gathered}
\int d \mu(g) \square \otimes \square^{\dagger}=\int d \mu(g) \square \otimes \square \square^{\dagger}=0, \\
\int d \mu(g) \square \square \otimes \square \square^{\dagger} \sim \mathbb{1}_{\square}, \quad \int d \mu(g) \square \otimes \square^{\dagger} \sim \mathbb{1}_{\square} .
\end{gathered}
$$

As we argued in Sec. III, the effective apparatus, given by the actual one and the lack of knowledge about it, is covariant [with respect to $\mathrm{SU}(d)$ in this case]. In terms of the Kraus operators, associated with measurement outcomes that do not transform upon a unitary rotation of the apparatus (e.g., LEDs or dials on a display), this means there is a unitary freedom in the next observers' possible knowledge of those Kraus operators for any given outcome, and an average is performed over $\mathrm{SU}(d)$. We restrict our attention to measurements with a single term in the Kraus decomposition for any outcome-see the discussion in Sec. V A.

Moreover, we assume that a given observer does not know the measurement outcomes of the previous observers, thus, no other object, except the $i$ th observer's output state, its probability, and guess, depends on the measurement outcome. Therefore, we can perform the sum over all outcomes to get the channel induced by such a measurement. Hence, one way to look at the measurement process is via the map,

$$
\hat{\rho} \mapsto \hat{\rho}^{\prime}=\chi(\hat{\rho})=\sum_{o} \int d \mu(g) g A_{o} g^{\dagger} \hat{\rho} g A_{o}^{\dagger} g^{\dagger},
$$

where $\{o\}$ is the set of possible outcomes of the preceding observer's apparatus (or the set enriched by additional outcomes so that a quantum operation performed given any outcome $o$ has a single-Kraus operator in its Kraus decomposition).

Using Eq. (C1), we get

$$
\chi(\hat{\rho})=\frac{c-1}{(d+1)(d-1)} \hat{\rho}+\frac{d^{2}-c}{(d+1)(d-1)} \frac{\mathbb{1}}{d},
$$

where

$$
c=\sum_{o}\left|\operatorname{Tr} A_{o}\right|^{2}
$$


[1] C. A. Fuchs and A. Peres, Phys. Today 53(3), 70 (2000).

[2] S. Gleyzes, S. Kuhr, C. Guerlin, J. Bernu, S. Deleglise, U. Busk Hoff, M. Brune, J.-M. Raimond, and S. Haroche, Nature (London) 446, 297 (2007).

[3] J. M. Raimond, M. Brune, and S. Haroche, Rev. Mod. Phys. 73, 565 (2001)

[4] M. Riebe, H. Haffner, C. F. Roos, W. Hansel, J. Benhelm, G. P. T. Lancaster, T. W. Korber, C. Becher, F. Schmidt-Kaler, D. F. V. James, and R. Blatt, Nature (London) 429, 734 (2004).

[5] D. Leibfried, R. Blatt, C. Monroe, and D. Wineland, Rev. Mod. Phys. 75, 281 (2003).

[6] V. Bužek, P. L. Knight, and N. Imoto, Phys. Rev. A 62, 062309 (2000).

[7] W. H. Zurek, Phys. Today 44(10), 36 (1991).

[8] R. Derka, V. Bužek, and A. K. Ekert, Phys. Rev. Lett. 80, 1571 (1998).

[9] K. Banaszek, Phys. Rev. Lett. 86, 1366 (2001).

[10] S. Massar and S. Popescu, Phys. Rev. Lett. 74, 1259 (1995).

[11] N. Gisin and S. Popescu, Phys. Rev. Lett. 83, 432 (1999).

[12] E. Bagan, M. Baig, A. Brey, R. Muñoz-Tapia, and R. Tarrach, Phys. Rev. Lett. 85, 5230 (2000).

[13] A. Peres and P. F. Scudo, Phys. Rev. Lett. 87, 167901 (2001).

[14] E. Bagan, M. Baig, and R. Muñoz-Tapia, Phys. Rev. Lett. 87, 257903 (2001).

[15] S. D. Bartlett, T. Rudolph, and R. W. Spekkens, Rev. Mod. Phys. 79, 555 (2007).

[16] E. B. Davies and J. T. Lewis, Commun. Math. Phys. 17, 239 (1970).
[17] A. S. Holevo, Probabilistic and Statistical Aspects of Quantum Theory, North-Holland Series in Statistics and Probability, Vol. 1 (North-Holland, Amsterdam, 1982).

[18] P. Rapčan, J. Calsamiglia, R. Muñoz-Tapia, E. Bagan, and V. Bužek, Phys. Scr. 2010, 014059 (2010).

[19] E. Bagan, M. Baig, A. Brey, R. Muñoz-Tapia, and R. Tarrach, Phys. Rev. A 63, 052309 (2001).

[20] J. I. Latorre, P. Pascual, and R. Tarrach, Phys. Rev. Lett. 81, 1351 (1998).

[21] A. Acín, J. I. Latorre, and P. Pascual, Phys. Rev. A 61, 022113 (2000).

[22] J.-C. Boileau, L. Sheridan, M. Laforest, and S. D. Bartlett, J. Math. Phys. 49, 032105 (2008).

[23] E. B. Davies, Quantum Theory of Open Systems (Academic, London, 1976).

[24] A. S. Holevo, J. Math. Phys. 39, 1373 (1998).

[25] M. G. Genoni and M. G. A. Paris, J. Phys.: Conf. Ser. 67, 012029 (2007).

[26] E. Bagan, A. Monras, and R. Muñoz-Tapia, Phys. Rev. A 71, 062318 (2005).

[27] L. Mišta and J. Fiurášek, Phys. Rev. A 74, 022316 (2006).

[28] M. Sabuncu, L. Mišta, J. Fiurášek, R. Filip, G. Leuchs, and U. L. Andersen, Phys. Rev. A 76, 032309 (2007).

[29] S. D. Bartlett, T. Rudolph, R. W. Spekkens, and P. S. Turner, New J. Phys. 8, 58 (2006).

[30] D. Poulin and J. Yard, New J. Phys. 9, 156 (2007).

[31] P. Rapčan, Ph.D. thesis, Comenius University, 2011. 